\title{
Raman Vibrational Shifts of Small Clusters of Hydrogen Isotopologues ${ }^{\dagger}$
}

Matthew Schmidt, $\ddagger$ José M. Fernández, "Nabil Faruk, $\ddagger$ Marcel Nooijen, $\stackrel{\ddagger}{\ddagger}$ Robert J. Le Roy, $\ddagger$ Juan H. Morilla, " Guzmán Tejeda, Salvador Montero, " and Pierre-Nicholas Roy,

$\ddagger$ Department of Chemistry, University of Waterloo, Waterloo, Ontario, Canada, N2L 3G1 \Laboratory of Molecular Fluid Dynamics, Instituto de Estructura de la Materia, CSIC, Serrano 121, E-28006 Madrid, Spain

E-mail: pnroy@uwaterloo.ca

\footnotetext{
${ }^{\dagger}$ DOI: 10.1021 /acs.jpca.5b08852

Journal of Physical Chemistry A 119, 12551-12561 (2015)

Special Issue: Dynamics of Molecular Collisions XXV: Fifty Years of Chemical Reaction Dynamics Received: September 10, 2015

Revised October 30, 2015

Published: October 30, 2015
} 


\begin{abstract}
Raman vibrational shifts of small parahydrogen $\left(\mathrm{pH}_{2}\right)$, orthodeuterium $\left(\mathrm{oD}_{2}\right)$, and paratritium $\left(\mathrm{pT}_{2}\right)$ clusters with respect to the free molecules are calculated by combining a first order perturbation theory approach with Langevin equation Path Integral Ground State (LePIGS) simulations [J. Phys. Chem. A 117, 7461 (2013)]. Our theoretical predictions are compared to existing cryogenic free jet expansion results for pure $\left(\mathrm{pH}_{2}\right)_{N}$ clusters [Phys. Rev. Lett. 92, 223401 (2004)] and to new measurements for $\left(\mathrm{oD}_{2}\right)_{N}$ clusters reported here. This method has been successfully used before to predict the Raman vibrational shifts of $\left(\mathrm{pH}_{2}\right)_{N}$ clusters [J. Chem. Phys. 141, 014310 (2014)]. The 6-D interaction potential of Hinde [J. Chem. Phys. 128, 154308 (2008)] is reduced to 1-D using the Adiabatic Hindered Rotor approximation to yield effective pair potentials for both molecules being in the ground vibrational state, and for one of them carrying one quantum of vibrational excitation. These reduced 1-D potentials are fitted to a Morse Long Range analytic form for later convenience. Good agreement between experiment and theory is found for the smaller clusters, but significant deviations remain for the larger ones.
\end{abstract}

\title{
1 Introduction
}

The study of hydrogen clusters has been the subject of much research in both theory and experiment; a key reason being that superfluid properties of parahydrogen have been observed

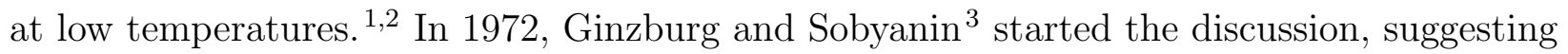
that $\mathrm{pH}_{2}$ may be a superfluid and proposed several ways to observe it. The next major theoretical breakthrough was in 1991, as Sindzingre et $a l^{4}$ calculated the superfluid fractions of $\mathrm{pH}_{2}$ clusters using Path Integral Monte Carlo (PIMC) and observed that below $2 \mathrm{~K},\left(\mathrm{pH}_{2}\right)_{13}$ and $\left(\mathrm{pH}_{2}\right)_{18}$ were superfluid, but $\left(\mathrm{pH}_{2}\right)_{33}$ remained non-superfluid. Despite supercooling liquid hydrogen to temperatures as low as $5.0 \mathrm{~K}$ experimentalists were unable to observe superfluidity experimentally, ${ }^{5-8}$ until Grebenev et $a l^{9}$ in 2000 provided the first experimental 
evidence. They measured the IR spectra of $\mathrm{OCS}\left(\mathrm{pH}_{2}\right)_{14,15,16}$ embedded in mixed ${ }^{3} \mathrm{He} /{ }^{4} \mathrm{He}$ droplets and observed that the Q-branch was not present when reducing the temperature from $0.38 \mathrm{~K}$ to $0.15 \mathrm{~K}$. This indicated that the contribution of the $\mathrm{pH}_{2}$ to the moment of inertia of the OCS molecule decreased to zero, which is representative of superfluidity since the OCS molecule was able to freely rotate within the parahydrogen cluster. Since then, many theoretical studies have looked at superfluidity of parahydrogen clusters around different dopant molecules. ${ }^{10-14}$ Joint experimental and theoretical work has recently allowed the first measurement of the so-called superfluid fraction of parahydrogen clusters. ${ }^{15,16}$ In addition to this, the solid-like or liquid-like nature of parahydrogen at low temperatures, including the zero temperature limit, have been extensively studied ${ }^{17-26}$ and discrepancies in the shape of the theoretically calculated ground state chemical potential have been discovered. Despite experimental challenges, in 2004 Tejeda et $a l^{27}$ produced small, pure parahydrogen clusters resolved in space and time using cryogenic free-jet expansions and measured the Raman vibrational spectra of sizes $N=2-8,13$, and 33 . They also provided a theoretical method based on first order perturbation theory (PT) for calculating the Raman vibrational shift for a cluster of $N$ identical particles using the pair distribution function, $P_{N}(R)$, and the 'difference potential', $\Delta V(R)$, defined as the difference between the potential energy surface when the system has one quantum of vibration and the potential energy surface when the system has zero quantum of vibration,

$$
\Delta \nu_{N}=(N-1) \int_{0}^{\infty} \Delta V(R) P_{N}(R) d R
$$

Using this formula, Diffusion Monte Carlo (DMC) calculations using the Buck interaction potential (B-P) were used to generate the ground state $P_{N}(R)$. Using the experimental spectra from $N=2-8$, the $\Delta V(R)$ was empirically fit to a Lennard-Jones form to reproduce the vibrational frequency shifts measured in that range and then predict the shifts for $N=13$ and 33 with good agreement. 
In 2014, Faruk and co-workers ${ }^{28}$ calculated the Raman vibrational shifts of $\left(\mathrm{pH}_{2}\right)_{N}$ clusters using the expression above. A crucial difference was however that the $\Delta V(R)$ was obtained from first-principles rather than an empirical fit to experiment as in Ref. 27. This meant that vibrational shifts could actually be predicted without resorting to prior experimental knowledge. Ground state quantum molecular dynamics simulations were performed using the Langevin equation Path Integral Ground State (LePIGS) method ${ }^{26,29}$ to generate the ground state $P_{N}(R)$ distributions. For these simulations, the 6-D Hinde potential (H6PES), ${ }^{30}$ a mostly $a b$ initio potential was reduced to $1-\mathrm{D}$ both in the ground vibrational state (H1-P) and with one vibrational quantum using the Adiabatic Hindered Rotor (AHR) approximation. ${ }^{31}$ The vibrational shifts were also calculated from simulations using the B-P ${ }^{32}$ and the Silvera-Goldmann (SG-P) ${ }^{33}$ potentials both empirically based functions. Comparisons with experimental data showed that the 1-D version of the H1-P potential outperforms the empirically based potentials for small cluster sizes $(N=2-8,13)$, though the Buck potential appears to give better results for the last experimental point at cluster size $N \simeq 33$. Most importantly, the shifts of Ref. 28 were predicted independently from experiment, a key difference from the work of Ref. 27.

In the present work, the H6-PES is reduced to 1-D ground state pair potentials, H1-P, for the other two isotopologues, $\mathrm{oD}_{2}$ and $\mathrm{pT}_{2}$, using the $\mathrm{AHR}$ approximation ${ }^{31}$ and appropriate internal vibrational averaging. The H1-Ps, in pairwise form, are used to calculate the Raman vibrational shifts using the LePIGS method plus the perturbation theory approach described above. We report new experimental values of the Raman vibrational shifts of small $\left(\mathrm{oD}_{2}\right)_{N}$ clusters for $N=2-6,9-11$. The predicted Raman vibrational shifts for $\left(\mathrm{oD}_{2}\right)_{N}$ clusters are compared to the new experimental results, while for $\left(\mathrm{pH}_{2}\right)_{N}$ clusters they are compared to earlier experimental results. ${ }^{27}$ The shifts predicted here using our H1-Ps are also compared to those calculated using distribution obtained from the empirically based, isotope-independent B-P.

It is important to note that our $\left(\mathrm{pH}_{2}\right)_{N}$ shifts calculated here are improved with respect 
to previous work. ${ }^{28}$ We use a denser mesh for our difference potential and our path integral simulations are performed at smaller imaginary timestep values, however we only notice minor differences in the third decimal place between the values reported previously, so they are still valid. A larger basis set is used here for the dimer and trimer calculations. Another new aspect is the quantification of the error associated with the AHR approximation and the error originating from first-order perturbation theory. We also introduce a way to scale our results to account for these errors. New results reported here include Raman shifts calculated: (i) from simulation for the dimer and trimer, (ii) using the $P_{N}(R)$ obtained from direct diagonalization as opposed to simulation for the dimer, and (iii) using direct diagonalization and second-order perturbation theory (PT-2) for the dimer.

Although all predicted shifts in this paper are calculated using the H1-Ps as a tabulated set of points in both the diagonalization and the simulation methods, we also fit these potentials to an analytic Morse Long Range (MLR) ${ }^{34,35}$ functional form denoted H1-MLR. Describing our potentials with Morse Long Range functional forms is not only useful due to their compactness, but also convenient for simulations since they have analytic derivatives.

The remainder of this paper is organized as follows: Theoretical and methodological details are described in Sec. 2, experimental vibrational shifts and their theoretical predictions are presented and discussed in Sec. 3, and we close with some concluding remarks and future direction in Sec. 4 .

\section{Theoretical Methodology}

\subsection{Calculating the Raman shifts for a cluster of identical particles}

The Raman vibrational shift for a cluster of $N$ identical particles is defined as the difference in vibrational excitation energy of the cluster and that of a free monomer. We focus on vibrational excitations from the ground state to a state with one total quantum of vibrational energy. It is possible to obtain an approximate pairwise-additive Hamiltonian along 
with first order perturbation theory to arrive at an expression for the Raman vibrational shift. Details are provided in the online supporting information where a simplified model is used to represent the energy levels for clusters of $N$ identical particles by constructing and diagonalizing the many-body Hamiltonian for the $v=0$ and $v=1$ vibrational states. The key result of that analysis is an expression for the difference potential $\Delta V(R)$ (defined as the difference between the energy of a pair of particles with 1 total quantum of vibration, and that of a pair with 0 total quantum of vibration). This vibrational shift can then be calculated using Eq. 1. The use of perturbation theory to calculate energy differences for a dopant (e.g. $\mathrm{CO}_{2}$ ) in a cluster of monomers (e.g. $\mathrm{H}_{2}$ ) has been widely used. ${ }^{10,13,36-38}$ The specific case when the dopant is identical to the monomers, leading to Eq. 1, was first considered in Ref. 27.

The quantity $\Delta V(R)$ that enters the theory can be determined explicitly by taking the difference between the ground state energy, $E_{0}$, and the symmetric excited vibrational state energy, $E_{+}$and is derived in the supporting information. The actual calculation of $E_{+}$and $E_{0}$ involves the construction of the full rovibrational Hamiltonian for the dimer. The energy $E_{+}$is that of the lowest symmetric state with one quantum of vibration. The full potential for a cluster of $N$ monomers with one quantum of vibration can be expressed in terms of the ground state potential $V(R)$ and the difference potential $\Delta V(R)$ as

$$
V_{N}^{\mathrm{v}=1}(\mathbf{R})=\sum_{i<j} V\left(R_{i j}\right)+\frac{2}{N} \sum_{i<j} \Delta V\left(R_{i j}\right)
$$

where the $2 / N$ factor comes from the restriction of counting distinct pairs. This provides a useful starting point for the discussion of perturbation theory later on.

\subsection{Constructing the effective H1-Ps for the dimer}

In practice the monomers within the cluster do not have a single internal degree of freedom. The exact space-fixed frame Hamiltonian for a cluster containing $N$ vibrating and rotating 
hydrogen molecules can be written as

$$
\hat{H}_{N}=\sum_{i}\left(\hat{h}_{i}^{\text {rovib }}+\hat{T}_{i}^{\text {trans }}\right)+\sum_{i<j} \hat{V}^{\text {aniso }}\left(r_{i}, r_{j}, R_{i}, R_{j}\right)
$$

where $r_{i}$ is the relative vector between the two atoms forming hydrogen molecule $i$, and $R_{i}$ is a vector representing the position of the center of mass of that molecule. The diatomic rovibrational Hamiltonian and translational kinetic energy of monomer $i$ are represented by $\hat{h}_{i}^{\text {rovib }}$ and $\hat{T}_{i}^{\text {trans }}$ respectively. The intermolecular interactions between molecules $i$ and $j$ are assumed to be pairwise additive and are represented by the $\hat{V}^{\text {aniso }}\left(r_{i}, r_{j}, R_{i}, R_{j}\right)$ term in the above expression. The superscript "aniso" is added to highlight the fact that the full anisotropy of the interaction is accounted for at this stage. The construction of the H1-P potential for $\mathrm{pH}_{2}$ was described in detail in a previous paper. ${ }^{28}$ The same method is used here to construct the $\mathrm{oD}_{2}-\mathrm{OD}_{2}$ and $\mathrm{pT}_{2}-\mathrm{pT}_{2}$ interaction potentials. The AHR method was developed by $\mathrm{Li}$ et $a l^{31}$ in studying the vibrational shifts of $\mathrm{CO}_{2}$ in $\left(\mathrm{pH}_{2}\right)_{N}$ clusters and has since been used for other systems including $\mathrm{H}_{2} \mathrm{O}-\mathrm{pH}_{2}{ }^{39}$ and $\mathrm{N}_{2} \mathrm{O}-\mathrm{pH}_{2} \cdot{ }^{40}$ For a dimer composed of two identical monomers, with a 6 -D interaction potential between them, the Hamiltonian in the space-fixed frame is

$$
\hat{H}=\hat{h}_{1}^{\text {rovib }}\left(r_{1}\right)+\hat{h}_{2}^{\text {rovib }}\left(r_{2}\right)+\hat{V}^{6-\mathrm{D}}\left(R, r_{1}, r_{2}, \theta_{1}, \theta_{2}, \phi\right)
$$

The AHR approach involves building the Hamiltonian at various distances, $R$, between molecules and diagonalizing over the 5 other degrees of freedom. For the ground state potential, the lowest eigenvalues calculated at each $R$ value are chosen resulting in a 1-D interaction potential. However, the individual monomer Hamiltonians $\hat{h}_{1}$ and $\hat{h}_{2}$ are not included as part of this AHR treatment, but instead the rovibrational energies (relative to the ground rotational state $J=0$ for each vibrational level) are used directly. The rovibrational radial wavefunctions are used as a basis set of primitive functions for constructing the Hamiltonian matrix. The quantum numbers $J=0,2,4$ are used for each monomer, 
reflecting the allowed rotational states for these molecules and symmetrization of the basis functions is incorporated as we are interested in the symmetric state, as discussed before. The basis functions are then converted into real spherical harmonics for convenience. For each system, one H1-P pair interaction potential is constructed with both monomers in their vibrational ground state and a second H1-P pair interaction potential is constructed with one quantum of vibration distributed between the two monomers. The so obtained energies for the ground and first excited vibrational states provide the potentials for the respective simulations.

The ground state Hamiltonian for the translational $N$-body problem then becomes

$$
\hat{H}_{g}=\hat{T}+\sum_{i<j} E_{0}\left(R_{i j}\right)
$$

where the ground state $E_{0}\left(R_{i j}\right)$ is composed of the individual intramolecular ground state energies $h_{0}$ and the intermolecular interaction potential $V(R)$

$$
E_{0}(R)=2 h_{0}+V(R)
$$

The Hamiltonian for the excited state then becomes

$$
\hat{H}_{e x c}=\hat{H}_{g}+\sum_{i<j} \Delta V\left(R_{i j}\right)
$$

where

$$
\Delta V\left(R_{i j}\right)=E_{+}\left(R_{i j}\right)-E_{0}\left(R_{i j}\right)
$$

and the energies for the excited state $E_{+}$and the ground state $E_{0}$ are obtained from the 6-D calculations for the dimer. In the simulations below, $\hat{H}_{g}$ is used to obtain the ground state density, while the perturbative correction [Eq. 1] is used to calculate the vibrational shift from the monomer. 


\subsection{Ground state simulation methodology}

The molecular dynamics simulations are performed using the Langevin equation Path Integral Ground State (LePIGS) $\operatorname{code}^{29}$ using the parameters and trial wavefunctions from a previous paper that focused on ground state calculations of hydrogen clusters. ${ }^{26}$ For simulations of cluster sizes $N \geq 4$, the Jastrow trial wavefunction, of form

$$
\psi_{T}=\exp \left\{-\frac{1}{2} \sum_{i<j}\left(\frac{b}{r_{i j}}\right)^{5}\right\}
$$

with parameter $b=3.65 \AA,{ }^{17}$ is used along with the corresponding parameters from the $N=4$ case from that paper. ${ }^{26}$ For $N=2-3$, the normal mode trial wavefunction, of form

$$
\psi_{T}=\exp \left\{-\frac{1}{2 \hbar} \sum_{k} \omega_{k} Q_{k}^{2}\right\}
$$

is used along with the corresponding parameters for the $N=4$ case, since it was determined that this trial wavefunction helps prevent the cluster from dissociating. The $Q_{k}$ 's represent the mass-weighted normal modes and the $\omega_{k}$ 's represent the angular frequencies determined from the eigenvalues and eigenvectors after diagonalizing the mass-weighted force constant matrix (Hessian). For each cluster size, starting with the initial conditions from the Cambridge Cluster Database, ${ }^{41}$ a steepest descent minimization is performed using the specific interaction potential to obtain the equilibrium geometries.

For each simulation, regardless of which trial wavefunction is implemented, a simulation time step $d t=5.0$ fs and an imaginary time step $\tau=0.001 \mathrm{~K}^{-1}$ are used. The initial conditions for all of our simulations come again from the Cambridge Cluster Database, ${ }^{41}$ and prior to our simulation that we sample from, a steepest descent minimization and an equilibration simulation of 25 ps are performed. 


\section{Results and Discussion}

\subsection{Experimental Raman vibrational shifts}

Raman scattering of small $\left(\mathrm{oD}_{2}\right)_{N}$ clusters has been recorded with the same experimental setup described in Ref. 42. In brief, $\left(\mathrm{oD}_{2}\right)_{N}$ clusters were formed in a supersonic free jet expansion by a continuous flow of a mixture of $\mathrm{oD}_{2}(10 \%)$ diluted in $\mathrm{He}$, at a stagnation pressure of $P_{0}=3$ bar into a very low pressure expansion chamber. The circular, thinwalled copper nozzle, $50 \mu \mathrm{m}$ in diameter, was cooled to $T_{0}=40 \mathrm{~K}$ by means of a closed cycle helium refrigerator. The temperature at the nozzle was actively controlled to $\pm 0.01 \mathrm{~K}$. The $\mathrm{oD}_{2}(10 \%)+$ He mixture was prepared in continuous flow by a pair of high-accuracy mass-flow controllers (Bronkhorst). The expansion chamber was evacuated by a 2000 litre/s turbomolecular pump, backed by a $400 \mathrm{~m}^{3} /$ hour roots blower and a $70 \mathrm{~m}^{3} /$ hour rotary pump. Background pressure in the expansion chamber was 0.006 mbar under the above given conditions.

$\mathrm{oD}_{2}$ was prepared from commercial normal $\mathrm{D}_{2}$ (PRAXAIR, nominal purity 99.8\%) by continuous flow through a catalytic converter operated at $21 \mathrm{~K}$ and 5 bar. The residence time under these conditions allowed the para-to-ortho conversion of the $\mathrm{D}_{2}$ molecules to reach the equilibrium ratio of $97.5 \%$ of $J=0$ molecules.

Raman scattering was excited by $4 \mathrm{~W}$ of a single-mode $\mathrm{Ar}^{+}$laser at $\lambda=514.5 \mathrm{~nm}$, sharply focused down to a $15 \mu \mathrm{m}$ beam waist onto the gas jet. The size of the probed volume seen by the detector of the spectrometer, is $90 \times 15 \times 2 \mu \mathrm{m}^{3}$. Raman scattering was collected at $90^{\circ}$ to both the gas jet and the exciting laser beam, by a high throughput $(f / 1.8)$ optical system with a total magnification $\times 10$, and was focused onto the $20 \mu \mathrm{m}$ entrance slit of the spectrometer, providing a $2 \mu \mathrm{m}$ spatial resolution along the jet axis. The spectrometer is equipped with a 2360 line/mm holographic $102 \times 102 \mathrm{~mm}^{2}$ grating and a back-illuminated CCD detector with $1340 \times 400$ pixels, cooled by liquid nitrogen to $-120{ }^{\circ} \mathrm{C}$. The spectral resolution was $0.16 \mathrm{~cm}^{-1}$. 
The Raman spectrum in Fig. 1 was recorded at $z=300 \mu \mathrm{m}$ from the nozzle orifice, at an estimated local temperature of $1.1 \mathrm{~K}$ in the jet. The peak at $2993.617 \mathrm{~cm}^{-1}$ is the $\mathrm{Q}(0)$ line ${ }^{43}$ of the free $\mathrm{oD}_{2}$ molecules, with an (off-scale) total signal of $\sim 75$ photons/s, while that at $2991.498 \mathrm{~cm}^{-1}$ is the $\mathrm{Q}(1)$ line due to the residual $2.5 \% \mathrm{pD}_{2}(J=1)$ molecules. The red-shifted satellite peaks at the bottom of the monomer $\mathrm{Q}(0)$ line are assigned to the excitation of one vibrational quantum in the small $\left(\mathrm{oD}_{2}\right)_{N}$ clusters, as was done for the small $\left(\mathrm{pH}_{2}\right)_{N}$ clusters. ${ }^{27,42,44}$ Peaks for $N=2$ to 6 are clearly resolved in the spectrum of Fig. 1, while those for $N=7$ and 8 are partially overlapped by the $\mathrm{Q}(1)$ line. The features redshifted from the $\mathrm{Q}(1)$ line are tentatively assigned to the $\left(\mathrm{oD}_{2}\right)_{N}$ clusters with $N=9$ to 11 , although they can also have small contribution from mixed $\mathrm{pD}_{2}-\mathrm{OD}_{2}$ dimers. The measured vibrational shifts of the $\left(\mathrm{oD}_{2}\right)_{N}$ clusters with respect to the free $\mathrm{oD}_{2}$ molecule are reported in Table 1.

Table 1: Raman vibrational shifts $\left(\right.$ in $\left.\mathbf{c m}^{-1}\right)$ of $\left(\mathrm{pH}_{2}\right)_{N}$ and $\left(\mathrm{oD}_{2}\right)_{N}$ clusters up to $N=11$ obtained from simulation $[\Delta \nu(\operatorname{Sim} \mid \mathbf{P T})]$ and new experimental values for $\left(\mathrm{oD}_{2}\right)_{N}$. Estimated uncertainties shown in parentheses. Those reported from simulation are standard errors using $1 \sigma$. Quantities marked with a $*$ are obtained from exact diagonalization $\left[\Delta \nu\left(\psi_{0} \mid \mathbf{P T}\right)\right]$. Results obtained using the $\mathbf{B}-\mathbf{P}^{\mathbf{3 2}}$ are also shown for comparison. The scaling factors used are 1.036 for $\mathrm{pH}_{2}$ and 1.030 for $\mathrm{oD}_{2}$. PT refers to first-order perturbation theory

\begin{tabular}{|c|c|c|c|c|c|c|c|c|}
\hline \multirow[b]{2}{*}{$N$} & \multicolumn{4}{|c|}{$\overline{\left(\mathrm{pH}_{2}\right)_{N}}$} & \multicolumn{4}{|c|}{$\left(\mathrm{oD}_{2}\right)_{N}$} \\
\hline & Expt. $^{27}$ & Scaled & $\overline{\mathrm{PT}}$ & B-P & Expt. & Scaled & $\mathrm{PT}$ & B-P \\
\hline 2 & $-0.400(20)$ & -0.40 & $-0.391^{*}$ & $-0.366(4)$ & $-0.401(30)$ & -0.41 & $-0.394^{*}$ & $-0.360(3)$ \\
\hline 3 & $-0.822(20)$ & -0.83 & $-0.801^{*}$ & $-0.756(4)$ & $-0.847(30)$ & -0.82 & $-0.795^{*}$ & $-0.731(3)$ \\
\hline 4 & $-1.251(20)$ & -1.25 & $-1.202(4)$ & $-1.107(5)$ & $-1.287(30)$ & -1.24 & $-1.202(3)$ & $-1.096(3)$ \\
\hline 5 & $-1.594(20)$ & -1.57 & $-1.518(4)$ & $-1.408(5)$ & $-1.602(30)$ & -1.51 & $-1.467(3)$ & $-1.341(3)$ \\
\hline 6 & $-1.910(20)$ & -1.86 & $-1.792(4)$ & $-1.641(5)$ & $-1.844(30)$ & -1.78 & $-1.724(3)$ & $-1.572(3)$ \\
\hline 7 & $-2.136(20)$ & -2.09 & $-2.016(4)$ & $-1.848(5)$ & & -1.99 & $-1.927(3)$ & $-1.756(3)$ \\
\hline 8 & $-2.350(20)$ & -2.28 & $-2.199(4)$ & $-2.025(5)$ & & -2.13 & $-2.063(3)$ & $-1.882(3)$ \\
\hline 9 & & -2.46 & $-2.372(4)$ & $-2.182(5)$ & $-2.457(30)$ & -2.28 & $-2.214(3)$ & $-2.021(3)$ \\
\hline 10 & & -2.62 & $-2.534(4)$ & $-2.327(5)$ & $-2.697(50)$ & -2.46 & $-2.383(3)$ & $-2.174(3)$ \\
\hline 11 & & -2.83 & $-2.729(4)$ & $-2.512(5)$ & $-2.827(50)$ & -2.61 & $-2.532(3)$ & $-2.307(3)$ \\
\hline
\end{tabular}




\subsection{Raman shifts of the dimers and trimers used to quantify method- ological errors and determine scaling factors}

To determine the Raman vibrational shifts from theory, we reduce the H6-PES to 1-D using the AHR approximation for a pair of monomers with zero total quanta of vibration, our H1-Ps, and with one total quantum of vibration and use a first-order perturbation approach [Eq. 1] to calculate the shifts. There are two advantages to doing these steps. First, it is clear that reducing the number of dimensions in our potential energy surface leads to computational savings. Also, it has been shown that the convergence of statistical errors with simulation time using our first-order perturbation approach is faster than simulating the cluster in each of its vibrational states and directly calculating the difference in energies to get the shift. ${ }^{38}$ For the dimer system of both $\mathrm{pH}_{2}$ and $\mathrm{oD}_{2}$, we directly calculate both the errors in the reduction from 6-D to 1-D and from using first-order perturbation theory instead of the direct method. We demonstrate the validity of using these two methods, as the cumulative error is smaller than that of experimental uncertainties. This allows us to propose a scaling factor applied to the entire cluster range to account for these two errors.

The ground state 1-D pair interaction potentials (H1-Ps) are calculated for the three $J=$ 0 isotopologues, $\mathrm{pH}_{2}, \mathrm{oD}_{2}$, and $\mathrm{pT}_{2}$ and are shown in Fig. 2. There are very small differences between them. As the mass increases, the minimum shifts towards shorter lengths and the well depth becomes shallower as the monomers become less polarizable. The difference potentials defined in the last section, are shown in Fig. 3. The difference potential becomes shallower as the mass increases since the vibrational energy levels become closer together. The ground state pair distribution functions from our simulations for cluster size $N=4$ are shown in Fig. 4. As the mass increases, the distributions become more peaked reflecting the reduced quantum character of the system.

For the dimer and trimer, in order to calculate the errors in the AHR and perturbation theory, we require the vibrational shifts to be calculated using diagonalization and simulations methods, using the H1-Ps. The most accurate method is to directly calculate the 
difference between the energy levels, denoted $\Delta \nu(\psi)$

$$
\Delta \nu(\psi)=E_{0}^{\mathrm{v}=1}-E_{0}^{\mathrm{v}=0}
$$

where $E_{0}$ represents the lowest eigenvalues obtained using the potential with one total quantum of vibration $(v=1)$ given by Eq. 2 or our H1-Ps with no excitation $(v=0)$. As mentioned in the previous section, perturbation theory can be applied to calculate the vibrational shifts by using Eq. 1. The ground state pair distribution function, $P_{N}(R)$ can be obtained through LePIGS simulations, and the shifts calculated using this method are denoted $\Delta \nu(\operatorname{Sim} \mid \mathrm{PT})$. However, from the diagonalizaton methods, we can calculate the pair distribution function directly from the ground state wavefunction, since $P_{N}(R)=\psi_{0}^{2}$. The vibrational shifts calculated this way are denoted $\Delta \nu\left(\psi_{0} \mid \mathrm{PT}\right)$.

Second-order perturbation theory ${ }^{45,46}$ can also be used to estimate the leading correction to the first-order perturbation theory method described above. When the wavefunction of interest is that of the ground state, the contribution

$$
\Delta \nu_{0, N}^{\prime \prime}=(N-1)^{2} \sum_{k \neq 0} \frac{\left|\left\langle\Phi_{k}^{(0)}|\Delta \hat{V}| \Phi_{0}^{(0)}\right\rangle\right|^{2}}{E_{0}^{(0)}-E_{k}^{(0)}}
$$

can be added to the $\Delta \nu$ of Eq. 1. The $k$ 's represent the excited vibrational states of the system and the $\Phi^{(0)}$ 's and $E^{(0)}$ 's represent the eigenvectors (wavefunctions) and eigenvalues (energies) obtained by diagonalizing the Hamiltonian using the ground state interaction potential. It should be noted that this correction is negative or zero, meaning that calculating the Raman shifts using only first-order perturbation theory should give results that are smaller in magnitude than the true value of the shift. The shifts calculated using this method are denoted $\Delta \nu\left(\psi_{0} \mid \mathrm{PT}-2\right)$.

Using these direct diagonalization methods, it is important to recognize that the reference frame is the body-fixed frame, the system is in the $J=0$ state, and the energies we calculate are due to the intermolecular translational motion. In simulations, the reference frame is the 
space-fixed frame. In the analysis throughout this paper, it should be noted that the shifts are compared based on their magnitude. Since only red shifts are observed, a larger shift means one that is more negative.

We first start with the dimer, which can be discussed in more detail. An exact method can be used to calculate the vibrational shift by diagonalizing the Hamiltonian for the $v=0$ and $v=1$ states using a Discrete Variable Representation (DVR) basis. ${ }^{47}$ These results, together with the simulation results, experimental values, and Hinde's exact 6-D calculations are shown in Table 2. The parameters used in our diagonalization code are 10000 DVR grid points, $r_{\min }=0.1 \AA r_{\max }=20.0 \AA$ for $\left(\mathrm{pH}_{2}\right)_{2}$ and $\left(\mathrm{oD}_{2}\right)_{2}$ and $r_{\max }=15.0 \AA$ for $\left(\mathrm{pT}_{2}\right)_{2}$, and $J_{\max }=0$ is used for all isotopologues. We note that our bound state energies are relative to those of the two free monomers.

Table 2: Ground state energies and Raman vibrational shifts (in $\left.\mathbf{c m}^{-1}\right)$ of $\left(\mathrm{pH}_{2}\right)_{2}$, $\left(\mathrm{oD}_{2}\right)_{2}$, and $\left(\mathrm{pT}_{2}\right)_{2}$ as calculated using our H1-Ps. Comparison is made to Hinde's results using his H6-PES Hinde 6-D Exact], ${ }^{30}$ new experimental data for $\left(\mathrm{oD}_{2}\right)_{2}$, and previous experimental results for $\left(\mathrm{pH}_{2}\right)_{2} \cdot{ }^{27} \Delta \nu(\psi)$ refers to the method of using direct diagonalization to calculate the shift by directly taking the difference between the ground state energy $\left[E_{0}\left(\psi_{0}\right)\right]$ and the energy of the excited vibrational state. Using exact diagonalization, the shifts are calculated using first order perturbation theory $\left[\Delta \nu\left(\psi_{0} \mid \mathbf{P T}\right)\right]$ and second order perturbation theory $\left[\Delta \nu\left(\psi_{0} \mid \mathbf{P T}-2\right)\right]$. The shifts are also calculated using the pair distribution functions from simulation $[\Delta \nu(\operatorname{Sim} \mid \mathbf{P T})]$. Estimated uncertainties are shown in parentheses. Those reported from simulation are standard errors using $1 \sigma$.

\begin{tabular}{llll}
\hline \hline & $\left(\mathrm{pH}_{2}\right)_{2}$ & $\left(\mathrm{oD}_{2}\right)_{2}$ & $\left(\mathrm{pT}_{2}\right)_{2}$ \\
\hline$E_{0}\left(\psi_{0}\right)$ & -2.90213 & -6.78567 & -9.055 \\
$\Delta \nu($ Hinde 6-D Exact $)$ & -0.405 & -0.406 & $\mathrm{~N} / \mathrm{A}$ \\
$\Delta \nu(\psi)$ & -0.401 & -0.400 & -0.363 \\
$\Delta \nu\left(\psi_{0} \mid \mathrm{PT}\right)$ & -0.391 & -0.394 & -0.359 \\
$\Delta \nu($ Sim $\mid \mathrm{PT})$ & $-0.388(3)$ & $-0.400(2)$ & $-0.363(2)$ \\
$\Delta \nu\left(\psi_{0} \mid \mathrm{PT}-2\right)$ & -0.401 & -0.400 & -0.363 \\
$\Delta \nu($ Experiment $)$ & $-0.400(20)$ & $-0.401(30)$ & $\mathrm{N} / \mathrm{A}$ \\
\hline
\end{tabular}

The difference of our $\Delta \nu(\psi)$ from the $\Delta \nu$ (Hinde 6-D Exact) can be directly attributed to the error in using the AHR approximation and vibrational averaging to reduce our potential from 6-D to 1-D. These errors lead to shifts that are $0.004 \mathrm{~cm}^{-1}$ smaller for $\left(\mathrm{pH}_{2}\right)_{2}$ and 
$0.006 \mathrm{~cm}^{-1}$ smaller for $\left(\mathrm{oD}_{2}\right)_{2}$ than Hinde's 6 -D values. The difference in our $\Delta \nu\left(\psi_{0} \mid \mathrm{PT}\right)$ and $\Delta \nu(\psi)$ is the error in using perturbation theory for these predictions. We observe that this error leads to even smaller shifts by $0.01 \mathrm{~cm}^{-1}$ for $\left(\mathrm{pH}_{2}\right)_{2}, 0.006 \mathrm{~cm}^{-1}$ for $\left(\mathrm{oD}_{2}\right)_{2}$, and $0.004 \mathrm{~cm}^{-1}$ for $\left(\mathrm{pT}_{2}\right)_{2}$. These two effects might explain why our $\Delta \nu(\operatorname{Sim} \mid \mathrm{PT})$ predictions for larger clusters are smaller than experimentally observed. This error is greatly eliminated with second order perturbation theory, $\Delta \nu\left(\psi_{0} \mid \mathrm{PT}-2\right)$, as it reproduces the shift using our most accurate method, $\Delta \nu(\psi)$. As expected, the shifts obtained from first order perturbation theory, either from simulation, $\Delta \nu(\operatorname{Sim} \mid \mathrm{PT})$, or from the exact calculation, $\Delta \nu\left(\psi_{0} \mid \mathrm{PT}\right)$, are in good agreement with each other.

Since we know the error due to the AHR approximation and to perturbation theory are both in the same direction (cause smaller shifts), the predicted shifts calculated using the $P_{N}(R)$ from simulation $[\Delta \nu(\operatorname{Sim} \mid \mathrm{PT})]$ and from direct diagonalization $\left[\Delta \nu\left(\psi_{0} \mid \mathrm{PT}\right)\right]$ can be scaled to correct for these errors. Using the shifts calculated for the dimer, $\Delta \nu_{2}$, the scaling factor $f$ for $\left(\mathrm{pH}_{2}\right)_{N}$ and $\left(\mathrm{oD}_{2}\right)_{N}$, is calculated as the ratio:

$$
f^{H / D}=\frac{\Delta \nu_{2}(\text { Hinde } 6 \text {-D Exact })}{\Delta \nu_{2}(\mathrm{PT})} .
$$

For $\mathrm{pT}_{2}$, there is no Hinde 6 -D Exact calculation so we instead scale to our $[\Delta \nu(\psi)]$ value using Lanczos diagonalization which accounts only for the error in perturbation theory but not the error due to the AHR approximation.

$$
f^{T}=\frac{\Delta \nu_{2}(\psi)}{\Delta \nu_{2}(\mathrm{PT})}
$$

The vibrational shift for the trimers can also be calculated exactly and perturbatively using the Lanczos diagonalization in Pekeris co-ordinates. ${ }^{48-50}$ These shifts are shown in Table 3, along with the simulation and experimental results. For each of the isotopologues, 90 dimensionless Jacobi DVR basis functions were used over 15000 iterations with a $V_{\max }$ of $1000 \mathrm{~cm}^{-1}$ for all cases except for the Lanczos-Exact calculation with $\left(\mathrm{pH}_{2}\right)_{3}$ 
$\left(V_{\max }=1100 \mathrm{~cm}^{-1}\right)$. For $\left(\mathrm{pH}_{2}\right)_{3}$ and $\left(\mathrm{oD}_{2}\right)_{3}, r_{\max }=20 \AA$ and for $\left(\mathrm{pT}_{2}\right)_{3} r_{\max }=15 \AA$. The basis function parameters used for all isotopologues are $a=5$ and $b=0$ with symmetry label $\mathrm{A}_{1}$. The first observation is the error in perturbation theory, or the deviation of $\Delta \nu\left(\psi_{0} \mid \mathrm{PT}\right)$

Table 3: Raman vibrational shifts $\left(\right.$ in $\left.\mathrm{cm}^{-1}\right)$ of $\left(\mathrm{pH}_{2}\right)_{3},\left(\mathrm{oD}_{2}\right)_{3}$, and $\left(\mathrm{pT}_{2}\right)_{3}$ as calculated using our H1-Ps. Comparison is made to new experimental data for $\left(\mathrm{oD}_{2}\right)_{3}$, and previous experimental results for $\left(\mathrm{pH}_{2}\right)_{3} \cdot{ }^{27} \Delta \nu(\psi)$ refers to the method of using direct diagonalization to calculate the shift by directly taking the difference between the ground state energy and the energy of the excited vibrational state. The shifts are also calculated using first order perturbation theory with the pair distribution functions determined by the exact wavefunctions $\left[\Delta \nu\left(\psi_{0} \mid \mathbf{P T}\right)\right]$ or from the results of simulation $[\Delta \nu(\operatorname{Sim} \mid \mathbf{P T})]$. Estimated uncertainties are shown in parentheses. Those reported from simulation are standard errors using $1 \sigma$.

\begin{tabular}{llll}
\hline \hline & $\left(\mathrm{pH}_{2}\right)_{3}$ & $\left(\mathrm{oD}_{2}\right)_{3}$ & $\left(\mathrm{pT}_{2}\right)_{3}$ \\
\hline$\Delta \nu(\operatorname{Sim} \mid \mathrm{PT})$ & $-0.798(4)$ & $-0.792(3)$ & $-0.722(2)$ \\
$\Delta \nu\left(\psi_{0} \mid \mathrm{PT}\right)$ & -0.801 & -0.795 & -0.723 \\
$\Delta \nu(\psi)$ & -0.814 & -0.803 & -0.728 \\
$\Delta \nu($ Experiment $)$ & $-0.822(20)$ & $-0.847(30)$ & $\mathrm{N} / \mathrm{A}$ \\
\hline
\end{tabular}

from the non-perturbative exact calculation, $\Delta \nu(\psi)$, is $0.013 \mathrm{~cm}^{-1}$ for $\left(\mathrm{pH}_{2}\right)_{3}, 0.008 \mathrm{~cm}^{-1}$ for $\left(\mathrm{oD}_{2}\right)_{3}$, and $0.005 \mathrm{~cm}^{-1}$ for $\left(\mathrm{pT}_{2}\right)_{3}$. We also confirm the same values of the shifts are obtained using the two different perturbation theory methods, $\Delta \nu(\operatorname{Sim} \mid \mathrm{PT})$ and $\Delta \nu\left(\psi_{0} \mid \mathrm{PT}\right)$.

\subsection{Raman vibrational shifts for entire cluster range}

We now extend our study to larger clusters, where direct diagonalization methods can no longer be used. Our predicted Raman vibrational shifts for $\left(\mathrm{pH}_{2}\right)_{N}$ and $\left(\mathrm{oD}_{2}\right)_{N}$ obtained using the H1-Ps over the range $N=2-11$ are compared to experiment in Fig. 5. The numerical results are given in Table 1. The shifts predicted for $\left(\mathrm{pT}_{2}\right)_{N}$ are given in Table 4, and they are smaller than those for both $\left(\mathrm{pH}_{2}\right)_{N}$ and $\left(\mathrm{oD}_{2}\right)_{N}$ for all cluster sizes. Results based on the B-P are also calculated for comparison.

The following discussion is based on the scaled values. The shifts reported in Table 1, which were calculated with our H1-P for $\left(\mathrm{pH}_{2}\right)_{N}$ are in good agreement with the experimental 
Table 4: Raman vibrational shifts $\left(\right.$ in $\left.\mathbf{c m}^{-1}\right)$ of $\left(\mathbf{p T}_{2}\right)_{N}$ clusters up to $N=11$ obtained from simulation $[\Delta \nu(\operatorname{Sim} \mid \mathbf{P T})]$ using our numerical H1-Ps and difference potentials for $\left(\mathbf{p} \mathbf{T}_{2}\right)$ dimers. Estimated uncertainties, reported as standard errors using $1 \sigma$, are shown in parentheses. Quantities marked with a $*$ are obtained from exact diagonalization $\left[\Delta \nu\left(\psi_{0} \mid \mathbf{P T}\right)\right]$ using the H1-Ps. Results obtained using the B-P ${ }^{32}$ potential are also shown for comparison. The scaling factor used is $f^{T}$ $=1.011$ for $\mathrm{pT}_{2}$.

\begin{tabular}{cccc}
\hline \hline & \multicolumn{3}{c}{$\left(\mathrm{pT}_{2}\right)_{N}$} \\
\cline { 2 - 4 }$N$ & Scaled & PT & B-P \\
\hline 2 & -0.36 & $-0.359^{*}$ & $-0.329(2)$ \\
3 & -0.73 & $-0.723^{*}$ & $-0.670(2)$ \\
4 & -1.10 & $-1.092(2)$ & $-1.001(3)$ \\
5 & -1.34 & $-1.327(2)$ & $-1.223(3)$ \\
6 & -1.57 & $-1.552(2)$ & $-1.430(3)$ \\
7 & -1.77 & $-1.755(2)$ & $-1.611(3)$ \\
8 & -1.86 & $-1.840(2)$ & $-1.695(3)$ \\
9 & -2.04 & $-2.013(2)$ & $-1.842(2)$ \\
10 & -2.18 & $-2.157(2)$ & $-1.977(2)$ \\
11 & -2.28 & $-2.254(2)$ & $-2.069(2)$ \\
\hline
\end{tabular}

data and fall within the uncertainty for $N=2-5$. For $N \geq 6$, the predicted shifts are distinctly smaller than the observed ones. This may be due to a number of possible factors. First, we introduce an ad hoc scaling factor for all cluster sizes based on the dimer to eliminate the effect of the AHR approximation and first order perturbation theory. Future work could be done to find a theoretically justified method to properly account for the errors due to those effects. Second, Hinde's 6-D potential also uses the same long range coefficients for $\mathrm{D}_{2}$ as it does for $\mathrm{H}_{2}$. This may contribute further to the deviation in the shifts of $\left(\mathrm{oD}_{2}\right)_{N}$ clusters between our calculated shifts and experiment. Finally, we are using pair interaction potentials and many-body effects may be important for the larger clusters.

It is also interesting to compare the relative magnitude of the vibrational shifts for $\left(\mathrm{pH}_{2}\right)_{N}$ and $\left(\mathrm{oD}_{2}\right)_{N}$. For $N \leq 5$, no difference in the experimental vibrational shift between $\left(\mathrm{pH}_{2}\right)_{N}$ and $\left(\mathrm{oD}_{2}\right)_{N}$ is observed within the estimated uncertainty, while for larger clusters, the experimental shifts for $\left(\mathrm{pH}_{2}\right)_{N}$ are larger than those for $\left(\mathrm{oD}_{2}\right)_{N}$. In turn, the ordering of the calculated shifts for $\left(\mathrm{pH}_{2}\right)_{N}$ and $\left(\mathrm{oD}_{2}\right)_{N}$ agrees with experiment; with perhaps the exception 
of $N=5$, since $\left(\mathrm{pH}_{2}\right)_{5}$ is predicted to have a larger shift than $\left(\mathrm{oD}_{2}\right)_{5}$ by $0.06 \mathrm{~cm}^{-1}$ while the shifts are experimentally observed to be almost equal.

For $N \leq 5$ small clusters of $\left(\mathrm{oD}_{2}\right)_{N}$ and $\left(\mathrm{pH}_{2}\right)_{N}$ we notice a competing effect in the calculation of the vibrational shift from perturbation theory, shown in Fig. 6, due to the shape and location of the pair distribution function with respect to the difference potential. This figure plots the integrand of the vibrational shift equation [Eq. 1] for the $\left(\mathrm{pH}_{2}\right)_{3}$, $\left(\mathrm{oD}_{2}\right)_{3}$, and $\left(\mathrm{pT}_{2}\right)_{3}$ trimers. The figure shows that $\left(\mathrm{oD}_{2}\right)_{3}$ has a significantly, narrowly peaked pair distribution in the most negative region of the difference potential contributing a very negative value to the shift. The $\left(\mathrm{pH}_{2}\right)_{3}$ pair distribution in the difference potential well is not as peaked and extends to longer distances, so although it does not contribute as negative a shift in the well region, since the distribution extends to longer distances it accumulates a more negative value. This leads to roughly equivalent values of the overall shift between $\left(\mathrm{pH}_{2}\right)_{N}$ and $\left(\mathrm{oD}_{2}\right)_{N}$.

For each isotopologue, the B-P gives consistently smaller vibrational shifts than our H1Ps and as a result the H1-Ps with isotopologue-dependence give better agreement for all cluster sizes.

\subsection{Portable analytic MLR models for our isotope-dependent H1- Ps}

Although the ground state H1-Ps used so far in this paper, both in the diagonalization and simulation methods, are the set of points obtained directly from the AHR reduction and vibrational averaging procedure, it is convenient to express our potentials in an analytic form. The potentials are fitted to the modern version of the MLR functional form: ${ }^{51}$

$$
V_{M L R}(r)=D_{e}\left\{1-\frac{u_{L R}(r)}{u_{L R}\left(r_{e}\right)} \exp \left[-\beta(r) \cdot y_{p}^{\mathrm{eq}}(r)\right]\right\}^{2}
$$


with $D_{e}$ representing the well depth and $r_{e}$ representing the equilibrium distance. The $\beta$ parameters are defined as:

$$
\beta_{M L R}(r)=y_{p}^{\mathrm{ref}}(r) \beta_{\infty}+\left[1-y_{p}^{r e f}(r)\right] \sum_{i=0}^{N_{\beta}} \beta_{i} y_{q}^{\mathrm{ref}}(r)^{i}
$$

in which

$$
\beta_{\infty}=\ln \left[\frac{2 D_{e}}{u_{L R}\left(r_{e}\right)}\right]
$$

and the $y_{a}$ 's are:

$$
y_{a}^{\mathrm{eq}}(r)=\frac{r^{a}-r_{e}^{a}}{r^{a}+r_{e}^{a}}
$$

and

$$
y_{a}^{\mathrm{ref}}(r)=\frac{r^{a}-r_{r e f}^{a}}{r^{a}+r_{r e f}^{a}}
$$

The long range contribution has the form:

$$
u_{L R}(r)=D_{m_{1}}(r) \frac{C_{m_{1}}}{r^{m_{1}}}+D_{m_{2}}(r) \frac{C_{m_{2}}}{r^{m_{2}}}+D_{m_{3}}(r) \frac{C_{m_{3}}}{r^{m_{3}}}+\ldots
$$

in which the $D_{m}(r)$ 's are "damping functions" from Refs. ${ }^{51,52}$

$$
D_{m_{i}}^{\mathrm{ds}}(r)=\left\{1-\exp \left(-\frac{3.30(\rho r)}{m_{i}}-\frac{0.423(\rho r)^{2}}{m_{i}^{1 / 2}}\right)\right\}^{m_{i}-1}
$$

The programs betaFIT ${ }^{53}$ and dpotFIT $^{54}$ are used to fit the H1-Ps to analytic Morse Long Range functions. To fit our potential in betaFIT, 756 points out of 17770 points were chosen from the original tabulated potential used in simulation. A required input to any sensible least squares fit program are "uncertainties" at each potential point. The uncertainties used here are a specific percentage of the value of the potential at each data point. The choice of points and uncertainties is listed in Table 5.

Our H1-MLRs are fitted to ten beta parameters and to optimize the remaining parameters they were fit using the betaFIT code program for a variety of different $p$ (between 5 and 8 ), 
Table 5: Selecting range and density of the tabulated H1-P data points to fit the analytic potential to and the choice of uncertainty percentages.

\begin{tabular}{ccc}
\hline \hline$R(\AA)$ & $\Delta R(\AA)$ & "Uncertainty" $(\%)$ \\
\hline $2.23-3.08$ & 0.05 & $10 \%$ \\
$3.10-8.08$ & 0.01 & $1 \%$ \\
$8.13-19.98$ & 0.05 & $10 \%$ \\
\hline
\end{tabular}

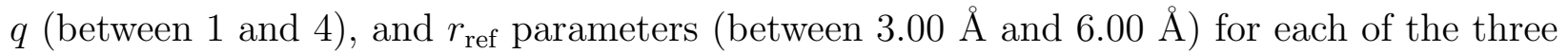
interaction potential surfaces. The parameters were chosen such that they minimize the dimensionless root mean squared deviation (so-called ' $d d$ ') between the fitted H1-MLRs and actual H1-Ps. The potFIT code was then used to further fine-tune the H1-MLRs, requiring that the dissociation limit be zero, which was not possible in betaFIT. The resulting ' $d d$ ' values for each potential were approximately 0.02 . The resulting potential surfaces obtained from potFIT are benchmarked by comparing the bound state energies of $\left(\mathrm{pH}_{2}\right)_{3},\left(\mathrm{oD}_{2}\right)_{3}$, and $\left(\mathrm{pT}_{2}\right)_{3}$ trimers obtained using our fitted H1-MLRs to those obtained using the true tabulated H1-P. It is most convenient to have the same MLR form $\left(p, q, r_{\text {ref }}\right.$ parameters) for each isotopologue and the parameters $p=6, q=1$, and $r_{\text {ref }}=4.20 \AA$ were chosen since these gave us the best overall results for each of the potential surfaces. The other MLR fitted parameters for each isotopologue are listed in Table 6. Comparing the H1-P to the H1-MLRs of the dimers in Table 7, the accuracy of the energies is on the order of the $0.0001 \mathrm{~cm}^{-1}$ and the errors in our calculated vibrational shifts are $0.002 \mathrm{~cm}^{-1}$, which are smaller than our simulated error bars. We find that the calculated ground state energies are the only bound states found using our H1-Ps and H1MLR-Ps for all three isotopologues. As mentioned before, the bound states refer only to the intermolecular translational degrees of freedom. Using his H6-PES, Hinde obtained for $\left(\mathrm{oD}_{2}\right)_{2}$ four bound states ${ }^{30}$ considering the end-over-end rotation of the dimer. The ground state wavefunctions for the $\left(\mathrm{pH}_{2}\right)_{2},\left(\mathrm{oD}_{2}\right)_{2}$, and $\left(\mathrm{pT}_{2}\right)_{2}$ dimers calculated from the exact code using our H1-Ps are shown in Fig. 7. It should be noted that the $\left(\mathrm{pH}_{2}\right)_{2}$ wavefunction is much broader than the other two. This reflects the greater quantum nature of $\left(\mathrm{pH}_{2}\right)_{2}$ compared to its heavier isotopologues. 
Table 6: MLR fit parameters for the ground state H1-Ps of $\mathrm{pH}_{2}, \mathrm{oD}_{2}$, and $\mathbf{p T}_{2}$. Energies are in $\mathbf{c m}^{-1}$ and lengths in $\AA$. Parameters common for all three H1-MLRs are $p=6, q=1, r_{\text {ref }}=4.60$, and $\rho^{A B}=1.10$.

\begin{tabular}{cccc}
\hline \hline Parameter & $\mathrm{pH}_{2}$ & $\mathrm{OD}_{2}$ & $\mathrm{pT}_{2}$ \\
\hline$D_{e}$ & 24.2288 & 24.2090 & 24.1595 \\
$r_{e}$ & 3.47005 & 3.46468 & 3.46043 \\
$V_{\text {min }}$ & -24.2288 & -24.2090 & -24.1595 \\
\hline$C_{6}$ & \multicolumn{3}{c}{$5.82036400 \mathrm{E}+04$} \\
$C_{8}$ & $2.87052154 \mathrm{E}+05$ \\
$C_{10}$ & \multicolumn{3}{c}{$1.80757343 \mathrm{E}+06$} \\
\hline$\beta_{0}$ & $-6.631 \mathrm{E}-02$ & $-6.396 \mathrm{E}-02$ & $-6.202 \mathrm{E}-02$ \\
$\beta_{1}$ & $1.346 \mathrm{E}-01$ & $2.109 \mathrm{E}-01$ & $2.795 \mathrm{E}-01$ \\
$\beta_{2}$ & $-3.300 \mathrm{E}-02$ & $4.970 \mathrm{E}-01$ & $9.750 \mathrm{E}-01$ \\
$\beta_{3}$ & $6.000 \mathrm{E}+00$ & $7.410 \mathrm{E}+00$ & $8.810 \mathrm{E}+00$ \\
$\beta_{4}$ & $-1.400 \mathrm{E}+01$ & $-1.570 \mathrm{E}+01$ & $-1.730 \mathrm{E}+01$ \\
$\beta_{5}$ & $-1.193 \mathrm{E}+02$ & $-1.020 \mathrm{E}+02$ & $-8.840 \mathrm{E}+01$ \\
$\beta_{6}$ & $2.290 \mathrm{E}+02$ & $4.110 \mathrm{E}+02$ & $5.880 \mathrm{E}+02$ \\
$\beta_{7}$ & $1.110 \mathrm{E}+03$ & $1.160 \mathrm{E}+03$ & $1.230 \mathrm{E}+03$ \\
$\beta_{8}$ & $-1.850 \mathrm{E}+03$ & $-3.150 \mathrm{E}+03$ & $-4.460 \mathrm{E}+03$ \\
$\beta_{9}$ & $-3.500 \mathrm{E}+03$ & $-3.300 \mathrm{E}+03$ & $-3.200 \mathrm{E}+03$ \\
$\beta_{10}$ & $6.000 \mathrm{E}+03$ & $1.100 \mathrm{E}+04$ & $1.600 \mathrm{E}+04$ \\
\hline
\end{tabular}

Table 7: Rotationless bound state energies and vibrational shifts (in $\mathrm{cm}^{-1}$ ) of $(\mathbf{p H})_{2},\left(\mathbf{o D}_{2}\right)_{2}$, and $\left(\mathbf{p} \mathbf{T}_{2}\right)_{2}$ dimers calculated using the Lanczos diagonalization method with our H1-P and the present H1-MLR. Energies are relative to the two free monomers.

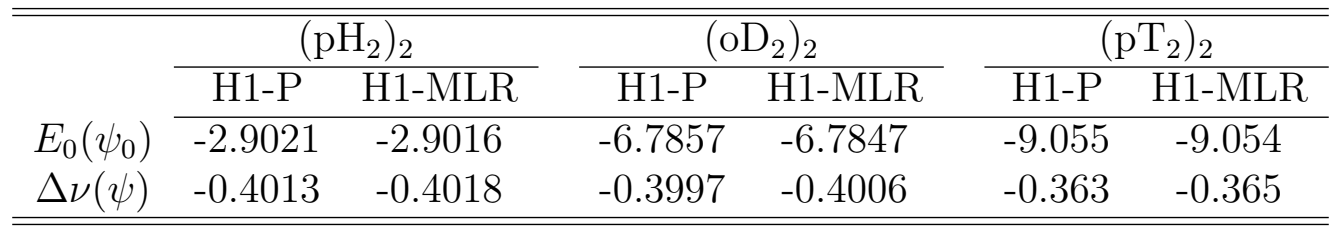

The results of the trimer bound state energy calculations, ${ }^{48,50}$ performed using the Lanczos diagonalization scheme, are shown in Table 8 using both the full pointwise H1-P and our fitted H1-MLR. We find two bound states for $\left(\mathrm{pH}_{2}\right)_{3}$, three for $\left(\mathrm{oD}_{2}\right)_{3}$ and four for $\left(\mathrm{pT}_{2}\right)_{3}$. We note that all bound states of the trimers lie below those of the dimers. Our fitted H1MLRs give good agreement with the original H1-Ps for the bound state calculations and the vibrational shifts. It is worth mentioning that similar calculations of the bound states of $\left(\mathrm{pH}_{2}\right)_{3}$ in the literature ${ }^{55,56}$ found only one bound state. This could be due to the interaction potential and future work should be done to determine the source of this discrepancy.

The one-dimensional particle distributions representing the wavefunctions in Pekeris coordinates are shown in Fig. 8 for the trimer of each isotopologue. The wavefunction for 
Table 8: Rotationless bound state energies $\mathrm{E}_{n}$ and vibrational shifts (in $\mathrm{cm}^{-1}$ ) of $\left(\mathbf{p H}_{2}\right)_{3},\left(\mathrm{oD}_{2}\right)_{3}$, and $\left(\mathbf{p} \mathrm{T}_{2}\right)_{3}$ trimers calculated using the Lanczos diagonalization method with our H1-P and the present H1-MLR. Energies are relative to the three free monomers.

\begin{tabular}{|c|c|c|c|c|c|c|}
\hline \multirow[b]{2}{*}{ Bound State Energies } & \multicolumn{2}{|c|}{$\left(\mathrm{pH}_{2}\right)_{3}$} & \multicolumn{2}{|c|}{$\left(\mathrm{oD}_{2}\right)_{3}$} & \multicolumn{2}{|c|}{$\left(\mathrm{pT}_{2}\right)_{3}$} \\
\hline & H1-P & H1-MLR & H1-P & H1-MLR & H1-P & H1-MLR \\
\hline$E_{0}$ & -9.8863 & -9.8864 & -21.2386 & -21.2388 & -27.905 & -27.904 \\
\hline$E_{1}$ & -3.65104 & -3.65098 & -12.7640 & -12.7634 & -18.076 & -18.075 \\
\hline$E_{2}$ & & & -7.2730 & -7.2727 & -13.923 & -13.922 \\
\hline$E_{3}$ & & & & & -9.483 & -9.485 \\
\hline$\Delta \nu\left(\psi_{0} \mid \mathrm{PT}\right)$ & -0.8010 & -0.8011 & -0.7954 & -0.7956 & -0.72272 & -0.72270 \\
\hline
\end{tabular}

the bound state $n=1$ level for the $\left(\mathrm{pH}_{2}\right)_{3}$ trimer is almost twice as wide as that for the ground state wavefunction. This extended wavefunction is not observed in the $\left(\mathrm{oD}_{2}\right)_{3}$ trimer, however a diffuse wavefunction is also observed in the $n=3$ level of the $\left(\mathrm{pT}_{2}\right)_{3}$ trimer that is much broader than the other three lower states.

\section{Concluding Remarks}

We have presented new experimental values for the Raman vibrational shifts for $\left(\mathrm{oD}_{2}\right)_{N}$ clusters of size $N=2-6$ and $9-11$. Effective 1-D pair interaction potentials for $\mathrm{pH}_{2}, \mathrm{oD}_{2}$, and $\mathrm{pT}_{2}$ have been constructed based on the H6-PES. ${ }^{30}$ Using these reduced pairwise H1-Ps, the Raman vibrational shifts were calculated using ground state quantum molecular dynamics (LePIGS) simulations and first order perturbation theory. For $\left(\mathrm{pH}_{2}\right)_{N}$ and $\left(\mathrm{oD}_{2}\right)_{N}$, the predictions using the H1-Ps are in good agreement with experimental results and outperform the traditional B-P model.

For $\left(\mathrm{pH}_{2}\right)_{2}$ and $\left(\mathrm{oD}_{2}\right)_{2}$ dimers, we estimated the errors associated with the reduction of the H6-PES to one dimensional models, along with the error originating from perturbation theory. Both sources of errors cause predicted shifts that are smaller in magnitude than expected. We find that second order perturbation theory corrects for the error of first order perturbation theory. A potential source of error for larger clusters is that the AHR reduction 
does not necessarily lead to a pairwise additive potential. Determining the quantitative effect of this error or if this assumption introduces many-body effects is a future research direction. For the trimers, we observe differing numbers of $J=0$ bound states of the trimer isotopologues: two for $\left(\mathrm{pH}_{2}\right)_{3}$, three for $\left(\mathrm{oD}_{2}\right)_{3}$, and four for $\left(\mathrm{pT}_{2}\right)_{3}$, however only one bound state for $\left(\mathrm{pH}_{2}\right)_{3}$ has been reported in the literature ${ }^{55,56}$ using methods similar to ours, and future work should be used to determine the source of that discrepancy.

Since the errors in the AHR approximation and perturbation theory are known, the simulation results can be scaled by a factor to account for the discrepancy. With this scaling, the calculated shifts of small clusters, $N \leq 5$, of $\left(\mathrm{pH}_{2}\right)_{N}$ and $\left(\mathrm{oD}_{2}\right)_{N}$ agree with experiment within estimated uncertainties, but they are smaller than experiment for larger clusters. This could be due to the simplified nature of the scaling approach. Also, the same long range coefficients are used for $\mathrm{D}_{2}$ as they are for $\mathrm{H}_{2}$ and this could lead to additional errors for the $\left(\mathrm{oD}_{2}\right)_{N}$ shifts. Future work could look at a theoretical method to properly account for the errors introduced by the AHR approximation and first-order perturbation theory, as well as trying to quantify the three-body or many-body effects to determine their impact on the vibrational shifts. For $\left(\mathrm{pT}_{2}\right)_{N}$, there are no experimental results, however a similar pattern is observed as for the other isotopologues that the H1-P gives larger shifts than the B-P for all cluster sizes. The predicted shifts for $\left(\mathrm{pT}_{2}\right)_{N}$ clusters are all smaller than the corresponding shifts for both $\left(\mathrm{pH}_{2}\right)_{N}$ and $\left(\mathrm{oD}_{2}\right)_{N}$.

Although the analysis above used the ground state H1-P as a set of points directly obtained from the AHR reduction, the H1-Ps were also fitted to a MLR ${ }^{34,35}$ functional form denoted H1-MLR. The dimer and trimer bound state energies and vibrational shifts with first order perturbation theory using the fitted H1-MLRs are compared with those using the H1-Ps directly and the results are in excellent agreement. Having an analytic form for the interaction potentials is very useful for quantum molecular dynamics simulations because they allow the derivatives (forces) to be calculated analytically. It also provides a compact way to represent the potential for dissemination purposes, instead of a tabulated form. 


\section{Acknowledgement}

This research has been supported by the Natural Sciences and Engineering Research Council of Canada (NSERC), the Canada Foundation for Innovation (CFI), and by the Spanish Ministerio de Economia y Competitividad (MINECO), through grants FIS2010-22064-C2 and FIS2013-48275-C2.

\section{Supporting Information Available}

A simplified model for the many-body problem, available at DOI: 10.1021/acs.jpca.5b08852. This material is available free of charge via the Internet at http://pubs.acs.org/.

\section{References}

(1) Zeng, T.; Roy, P.-N. Microscopic molecular superfluid response: theory and simulations. Rep. Prog. Phys. 2014, 77, 046601.

(2) Zeng, T.; Li, H.; Roy, P.-N. Potential generation and path-integral Monte Carlo in study of microscopic superfluidity. Int. J. Quant. Chem. 2015, 115, 535-540.

(3) Ginzburg, V.; Sobyanin, A. Can liquid molecular hydrogen be superfluid. JETP Lett. 1972, 15, 242.

(4) Sindzingre, P.; Ceperley, D. M.; Klein, M. L. Superfluidity in clusters of p- $\mathrm{H}_{2}$ molecules. Phys. Rev. Lett. 1991, 67, 1871.

(5) Maris, H.; Seidel, G.; Huber, T. Supercooling of liquid $\mathrm{H}_{2}$ and the possible production of superfluid $\mathrm{H}_{2}$. J. Low Temp. Phys 1983, 51, 471-487.

(6) Seidel, G.; Maris, H.; Williams, F.; Cardon, J. Supercooling of liquid hydrogen. Phys. Rev. Lett. 1986, 56, 2380. 
(7) Maris, H.; Seidel, G.; Williams, F. Experiments with supercooled liquid hydrogen. Phys. Rev. B 1987, 36, 6799 .

(8) Knuth, E.; Schünemann, F.; Toennies, J. Supercooling of $\mathrm{H}_{2}$ clusters produced in freejet expansions from supercritical states. J. Chem. Phys. 1995, 102, 6258-6271.

(9) Grebenev, S.; Sartakov, B.; Toennies, J. P.; Vilesov, A. F. Evidence for superfluidity in para-hydrogen clusters inside helium-4 droplets at 0.15 kelvin. Science 2000, 289, $1532-1535$.

(10) Kwon, Y.; Whaley, K. B. Nanoscale Molecular Superfluidity of Hydrogen. Phys. Rev. Lett. 2002, 89, 273401.

(11) Li, H.; Roy, P.-N.; Le Roy, R. J. Analytic Morse/long-range potential energy surfaces and predicted infrared spectra for $\mathrm{CO}_{2}-\mathrm{H}_{2}$. J. Chem. Phys. 2010, 132, 214309.

(12) Li, H.; Zhang, X.-L.; Le Roy, R. J.; Roy, P.-N. Analytic Morse/long-range potential energy surfaces and predicted infrared spectra for $\mathrm{CO}-\mathrm{H}_{2}$ dimer and frequency shifts of CO in $\left(\text { para- } \mathrm{H}_{2}\right)_{N} \mathrm{~N}=1-20$ clusters. J. Chem. Phys. 2013, 139, 164315.

(13) Zeng, T.; Li, H.; Roy, P.-N. Simulating Asymmetric Top Impurities in Superfluid Clusters: A para-Water Dopant in para-Hydrogen. J. Phys. Chem. Lett. 2013, 4, 18-22.

(14) Zeng, T.; Guillon, G.; Cantin, J. T.; Roy, P.-N. Probing the Superfluid Response of para-Hydrogen with a Sulfur Dioxide Dopant. J. Phys. Chem. Lett. 2013, 4, 2391-2396.

(15) Li, H.; Le Roy, R. J.; Roy, P.-N.; McKellar, A. A molecular superfluid: non-classical rotations in doped para-hydrogen clusters. Phys. Rev. Lett. 2010, 133401.

(16) Raston, P.; Jäger, W.; Li, H.; Le Roy, R.; Roy, P.-N. Persistent Molecular Superfluid Response in Doped Para-Hydrogen Clusters. Phys. Rev. Lett. 2012, 108, 253402.

(17) Cuervo, J. E.; Roy, P.-N. Path integral ground state study of finite-size systems: Application to small (parahydrogen $)_{N}(\mathrm{~N}=2-20)$ clusters. J. Chem. Phys. 2006, 125, 124314. 
(18) Cuervo, J. E.; Roy, P.-N. On the solid- and liquidlike nature of quantum clusters in their ground state. J. Chem. Phys. 2008, 128, 224509.

(19) Guardiola, R.; Navarro, J. A diffusion Monte Carlo study of small para-Hydrogen clusters. Cent. Eur. J. Phys. 2007, 6, 33-37.

(20) Khairallah, S. A.; Sevryuk, M. B.; Ceperley, D. M.; Toennies, J. P. Interplay between magic number stabilities and superfluidity of small parahydrogen clusters. Phys. Rev. Lett. 2007, 98, 4 .

(21) Mezzacapo, F.; Boninsegni, M. Structure, superfluidity, and quantum melting of hydrogen clusters. Phys. Rev. A 2007, 75, 033201.

(22) Mezzacapo, F.; Boninsegni, M. Classical and quantum physics of hydrogen clusters. J. Phys.: Condens. Matter 2009, 21, 164205-164211.

(23) Warnecke, S.; Sevryuk, M. B.; Ceperley, D. M.; Toennies, J. P.; Guardiola, R.; Navarro, J. The structure of para-hydrogen clusters. Eur. Phys. J. D 2010, 56, 353-358.

(24) Mezzacapo, F.; Boninsegni, M. On the Possible Supersolid Character of Parahydrogen Clusters. J. Phys. Chem. A 2011, 115, 6831-6837.

(25) Schmidt, M. Developing a Method to Study Ground State Properties of Hydrogen Clusters. M.Sc. thesis, University of Waterloo, 2014.

(26) Schmidt, M.; Constable, S.; Ing, C.; Roy, P. N. Inclusion of trial functions in the Langevin equation path integral ground state method: Application to parahydrogen clusters and their isotopologues. J. Chem. Phys. 2014, 140, 234101.

(27) Tejeda, G.; Fernández, J. M.; Montero, S.; Blume, D.; Toennies, J. P. Raman spectroscopy of small para- $\mathrm{H}_{2}$ clusters formed in cryogenic free jets. Phys. Rev. Lett. 2004, 92, 223401. 
(28) Faruk, N.; Schmidt, M.; Li, H.; Le Roy, R. J.; Roy, P.-N. First-principles prediction of the Raman shifts in parahydrogen clusters. J. Chem. Phys. 2014, 141, 014310.

(29) Constable, S.; Schmidt, M.; Ing, C.; Zeng, T.; Roy, P.-N. Langevin Equation Path Integral Ground State. J. Phys. Chem. A 2013, 117, 7461-7467.

(30) Hinde, R. J. A six-dimensional $\mathrm{H}_{2}-\mathrm{H}_{2}$ potential energy surface for bound state spectroscopy. J. Chem. Phys. 2008, 128, 154308.

(31) Li, H.; Roy, P.-N.; Le Roy, R. J. An "adiabatic-hindered-rotor" treatment allows para$\mathrm{H}_{2}$ to be treated as if it were spherical. J. Chem. Phys. 2010, 133, 104305.

(32) Buck, U.; Huisken, F.; Kohlhase, A.; Otten, D.; Schaefer, J. State resolved rotational excitation in $\mathrm{D}_{2}+\mathrm{H}_{2}$ collisions. J. Chem. Phys. 1983, 78, 4439-4450.

(33) Silvera, I. F.; Goldman, V. V. The isotropic intermolecular potential for $\mathrm{H}_{2}$ and $\mathrm{D}_{2}$ in the solid and gas phases. J. Chem. Phys. 1978, 69, 4209-4213.

(34) Le Roy, R. J.; Huang, Y.; Jary, C. An accurate analytic potential function for groundstate $\mathrm{N}_{2}$ from a direct-potential-fit analysis of spectroscopic data. J. Chem. Phys. 2006, $125,164310$.

(35) Le Roy, R. J.; Henderson, R. D. E. A new potential function form incorporating extended long-range behaviour: application to ground-state $\mathrm{Ca}_{2}$. Mol. Phys. 2007, 105, 663-677.

(36) Eichenauer, D.; Le Roy, R. J. Monte Carlo simulations of structural properties and infrared spectra of $\mathrm{SF}_{6}-(\mathrm{Ar})_{n}$ clusters. J. Chem. Phys. 1988, 88, 2898-2912.

(37) Chartrand, D. J.; Shelley, J. C.; Roy, R. J. L. Pulling, packing and stacking: structural proclivities of sulfur hexafluoride-(rare gas)n van der Waals clusters. J. Phys. Chem. 1991, 95, 8310-8328. 
(38) Li, H.; Blinov, N.; Roy, P.-N.; Le Roy, R. J. Path-integral Monte Carlo simulation of $\nu 3$ vibrational shifts for $\mathrm{CO}_{2}$ in $(\mathrm{He})_{n}$ clusters critically tests the $\mathrm{He}-\mathrm{CO}_{2}$ potential energy surface. J. Chem. Phys. 2009, 130, 144305.

(39) Zeng, T.; Li, H.; Le Roy, R. J.; Roy, P.-N. Adiabatic-hindered-rotor treatment of the parahydrogen-water complex. J. Chem. Phys. 2011, 135, 094304.

(40) Wang, L.; Xie, D.; Roy, R. J. L.; Roy, P.-N. A new six-dimensional potential energy surface for $\mathrm{H}_{2}-\mathrm{N}_{2} \mathrm{O}$ and its adiabatic-hindered-rotor treatment. J. Chem. Phys. 2013, 139, 034312 .

(41) Wales, D. J.; Doye, J. P. K. Global Optimization by Basin-Hopping and the Lowest Energy Structures of Lennard-Jones Clusters Containing up to 110 Atoms. J. Phys. Chem. A 1997, 101, 5111-5116.

(42) Morilla, J.; Fernández, J.; Tejeda, G.; Montero, S. The onset of molecular condensation: hydrogen. Physical Chemistry Chemical Physics 2010, 12, 12060-12064.

(43) Jennings, D. E.; Weber, A.; Brault, J. Raman spectroscopy of gases with a Fourier transform spectrometer: the spectrum of $\mathrm{D}_{2}$. Applied optics 1986, 25, 284-290.

(44) Montero, S.; Morilla, J. H.; Tejeda, G.; Fernández, J. M. Experiments on small $\left(\mathrm{H}_{2}\right)_{N}$ clusters. Eur. Phys. J. D 2009, 52, 31-34.

(45) Schrödinger, E. Quantisierung als Eigenwertproblem. Ann. Phys. 1926, 385, 437-490.

(46) Raleigh, J. W. Theory of Sound, 2nd ed.; London: Macmillan, 1894; pp 115-118.

(47) Colbert, D. T.; Miller, W. H. A novel discrete variable representation for quantum mechanical reactive scattering via the S-matrix Kohn method. J. Chem. Phys. 1992, 96, 1982-1991. 
(48) Roy, P.-N. Energy levels and wave functions of weakly bound bosonic trimers using Pekeris coordinates and a symmetry-adapted Lanczos approach. J. Chem. Phys. 2003, $119,5437-5443$.

(49) Liu, Y. D.; Roy, P.-N. Energy levels and wave functions of weakly-bound ${ }^{4} \mathrm{He}_{x}{ }^{20} \mathrm{Ne}_{y} \mathrm{H}$ $(\mathrm{x}+\mathrm{y}=2)$ systems using Pekeris coordinates and a symmetry-adapted Lanczos approach. J. Chem. Phys. 2004, 121, 6282-6289.

(50) Nightingale, M.; Roy, P.-N. Excited states of weakly bound bosonic clusters: Discrete variable representation and quantum Monte Carlo. J. Phys. Chem. A 2006, 110, 53915394.

(51) Le Roy, R. J.; Haugen, C. C.; Tao, J.; Li, H. Long-range damping functions improve the short-range behaviour of MLR potential energy functions. Mol. Phys. 2011, 109, $435-446$.

(52) Douketis, C.; Scoles, G.; Marchetti, S.; Zen, M.; Thakkar, A. J. Intermolecular forces via hybrid Hartree-Fock-SCF plus damped dispersion (HFD) energy calculations. An improved spherical model. J. Chem. Phys. 1982, 76, 3057-3063.

(53) Le Roy, R. J. betaFIT 2.1: A computer program to fit potential function points to selected analytic functions, University of Waterloo Chemical Physics Research Report No. CP-665; 2013.

(54) Le Roy, R. J.; Seto, J.; Huang, Y. DPotFit 2.0: A computer program for fitting diatomic molecule spectra to potential energy functions, University of Waterloo Chemical Physics Research Report CP-667; 2009.

(55) Yu, H.-G. Full-dimensional quantum calculations of vibrational spectra of six-atom molecules. I. Theory and numerical results. J. Chem. Phys. 2004, 120, 2270-2284. 
(56) Costa, L. S.; Clary, D. C. Calculation of the energy levels of weakly bound molecular trimers: Application to $\left(\mathrm{H}_{2}\right)_{3}$. J. Chem. Phys. 2002, 117, 7512-7519. 


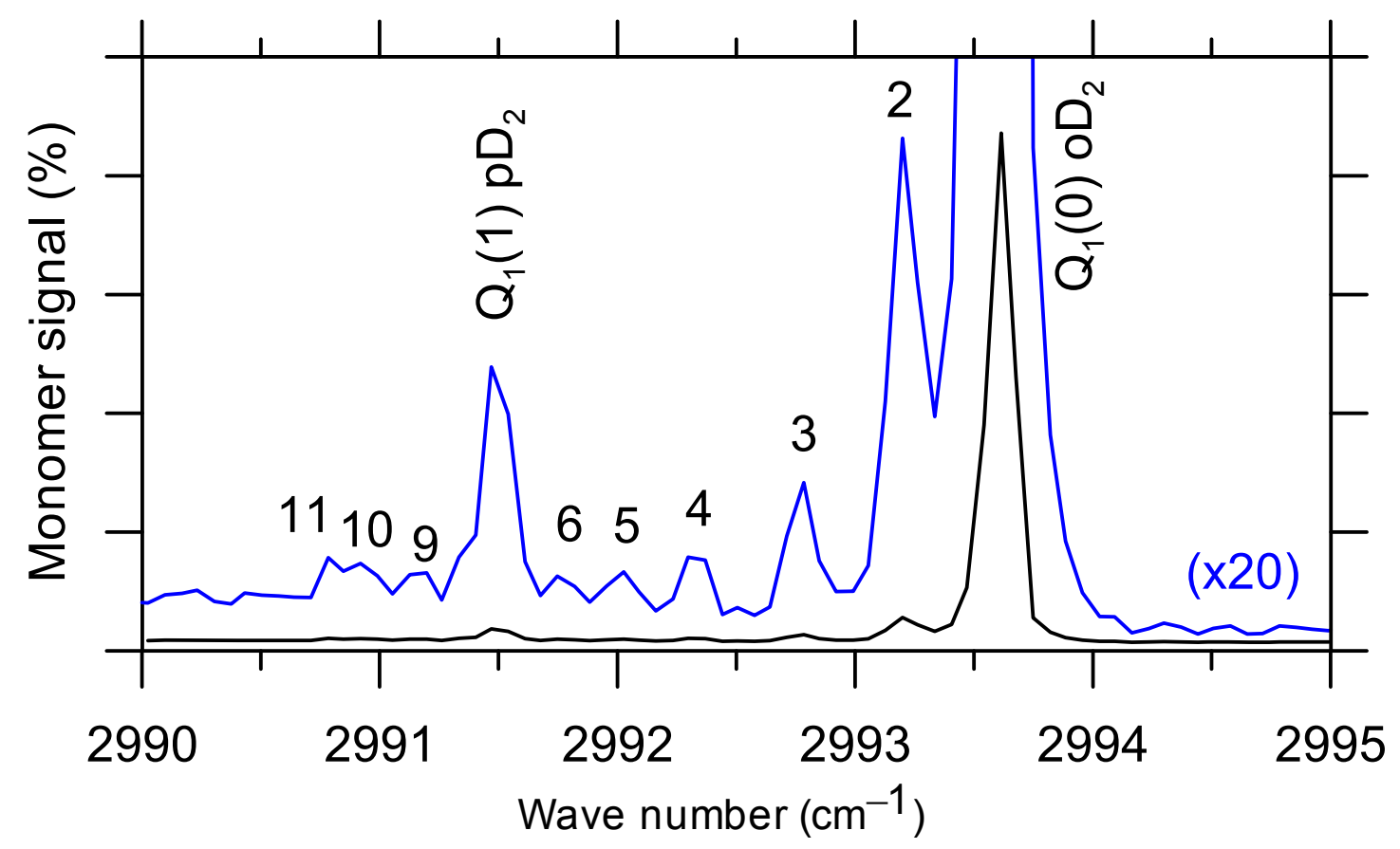

Figure 1: Vibrational Raman spectrum of small $\left(\mathrm{oD}_{2}\right)_{N}$ clusters, as recorded in a cryogenic free jet of $\mathrm{oD}_{2}(10 \%)+\mathrm{He}$ mixture. The numbers 2-11 indicate the size $N$ of the cluster. Experimental conditions (see text): $T_{0}=40 \mathrm{~K}, P_{0}=3 \mathrm{bar}, D=50 \mu \mathrm{m}, z=300 \mu \mathrm{m}$. The estimated local temperature is $1.1 \mathrm{~K}$. 


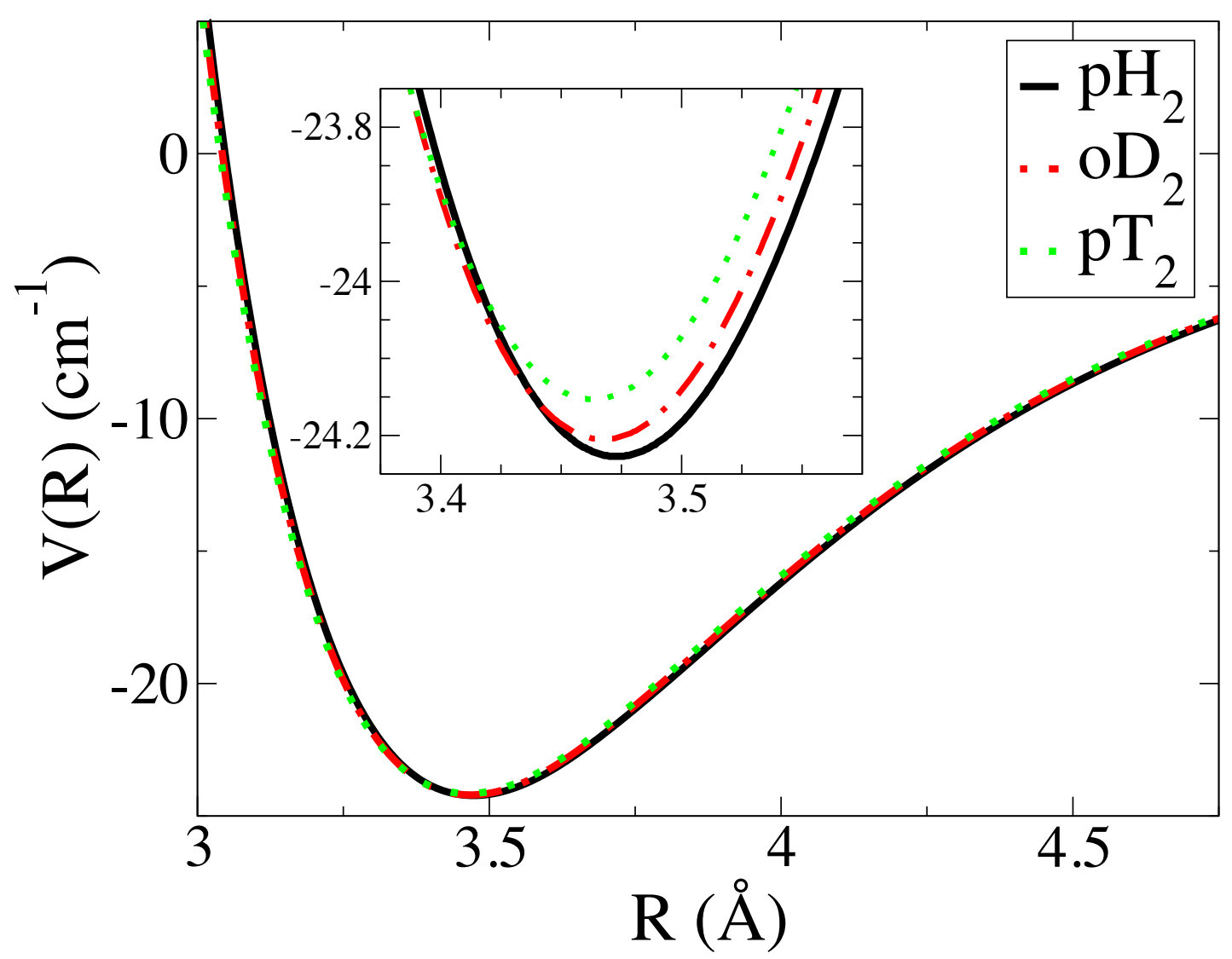

Figure 2: $\mathrm{H} 1-\mathrm{Ps}$ of $\mathrm{pH}_{2}, \mathrm{oD}_{2}$, and $\mathrm{pT}_{2}$ molecules in the ground $(v=0)$ vibrational state. 


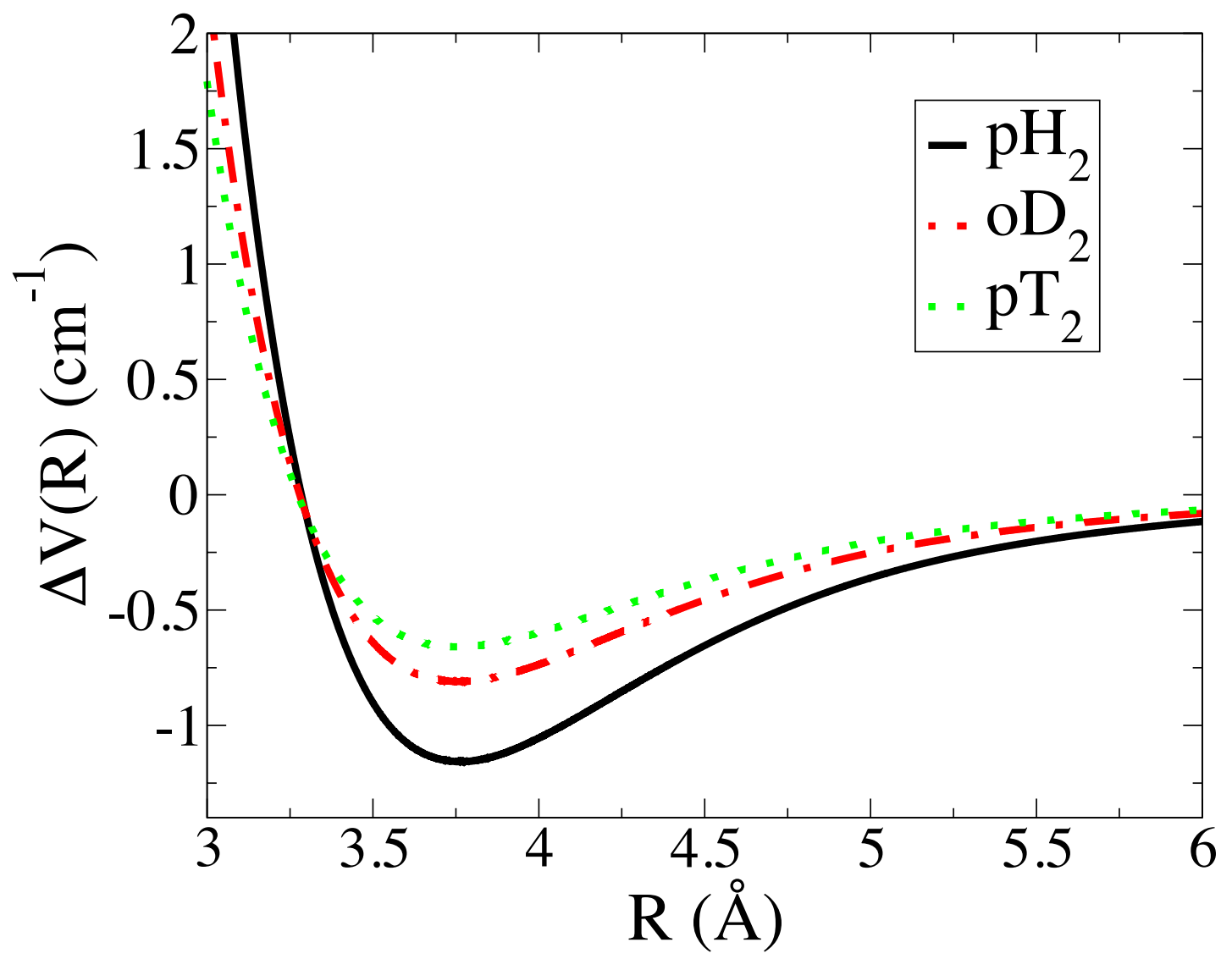

Figure 3: Difference between the H1-Ps of $\mathrm{pH}_{2}, \mathrm{oD}_{2}$, and $\mathrm{pT}_{2}$ molecules in the first excited $(v=1)$ and the ground $(v=0)$ vibrational states. 


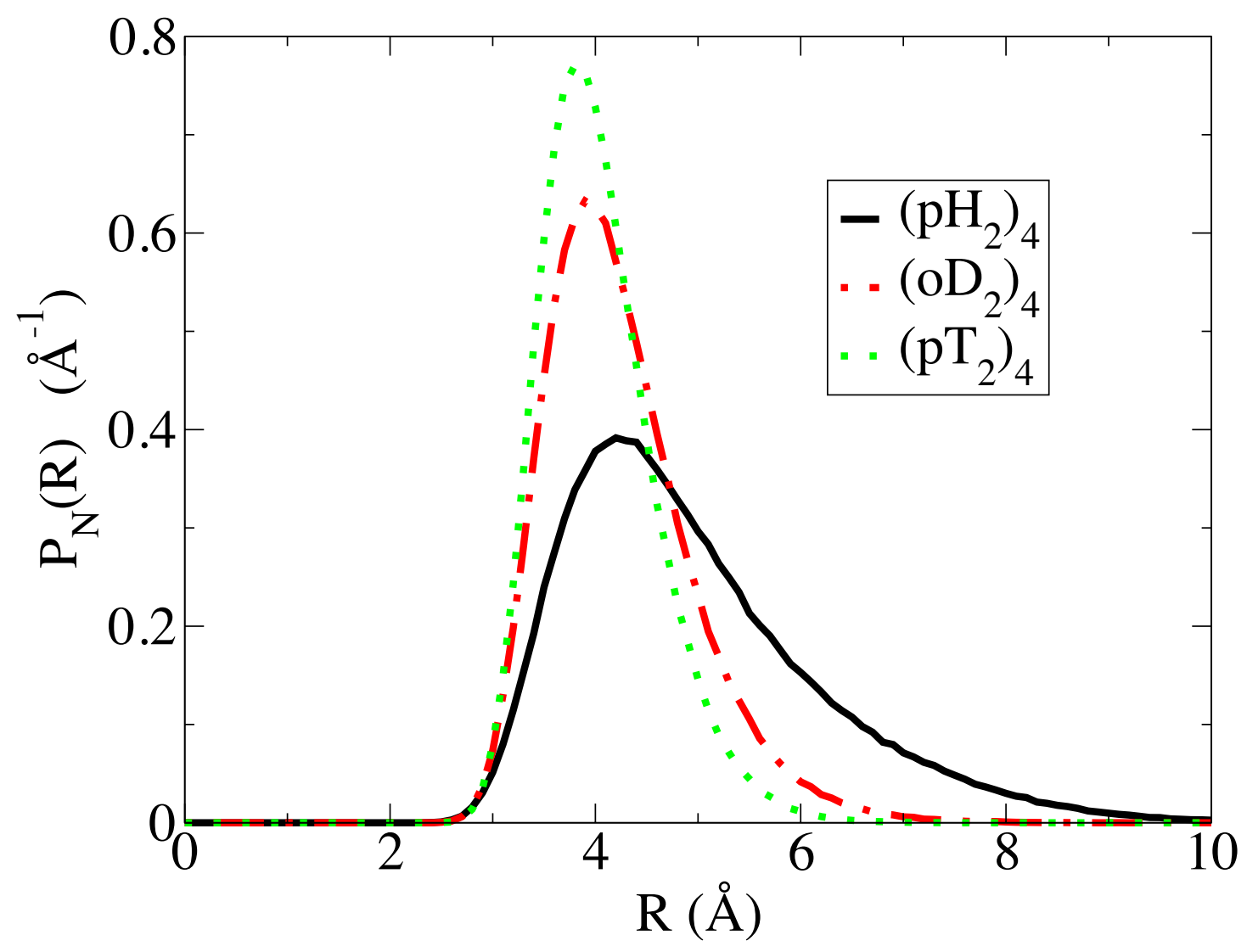

Figure 4: Pair distribution functions generated from LePIGS simulations of $\left(\mathrm{pH}_{2}\right)_{4},\left(\mathrm{oD}_{2}\right)_{4}$, and $\left(\mathrm{pT}_{2}\right)_{4}$ clusters using the H1-P for each isotopologue. 

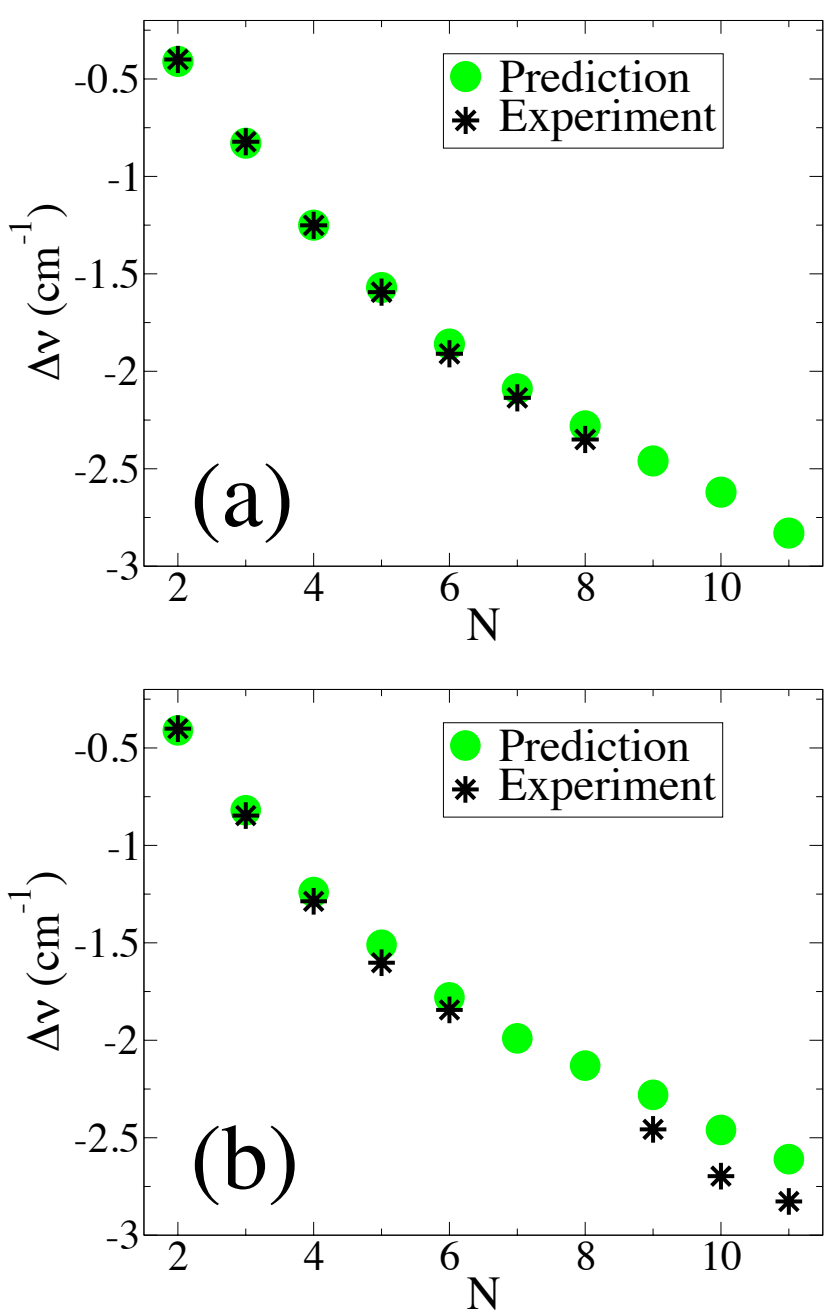

Figure 5: Predicted Raman vibrational shifts (with scaling) for (a) $\left(\mathrm{pH}_{2}\right)_{N}$ and (b) $\left(\mathrm{oD}_{2}\right)_{N}$ clusters up to size $N=11$ using the H1-P for each isotopologue. Experimental results are also shown. 


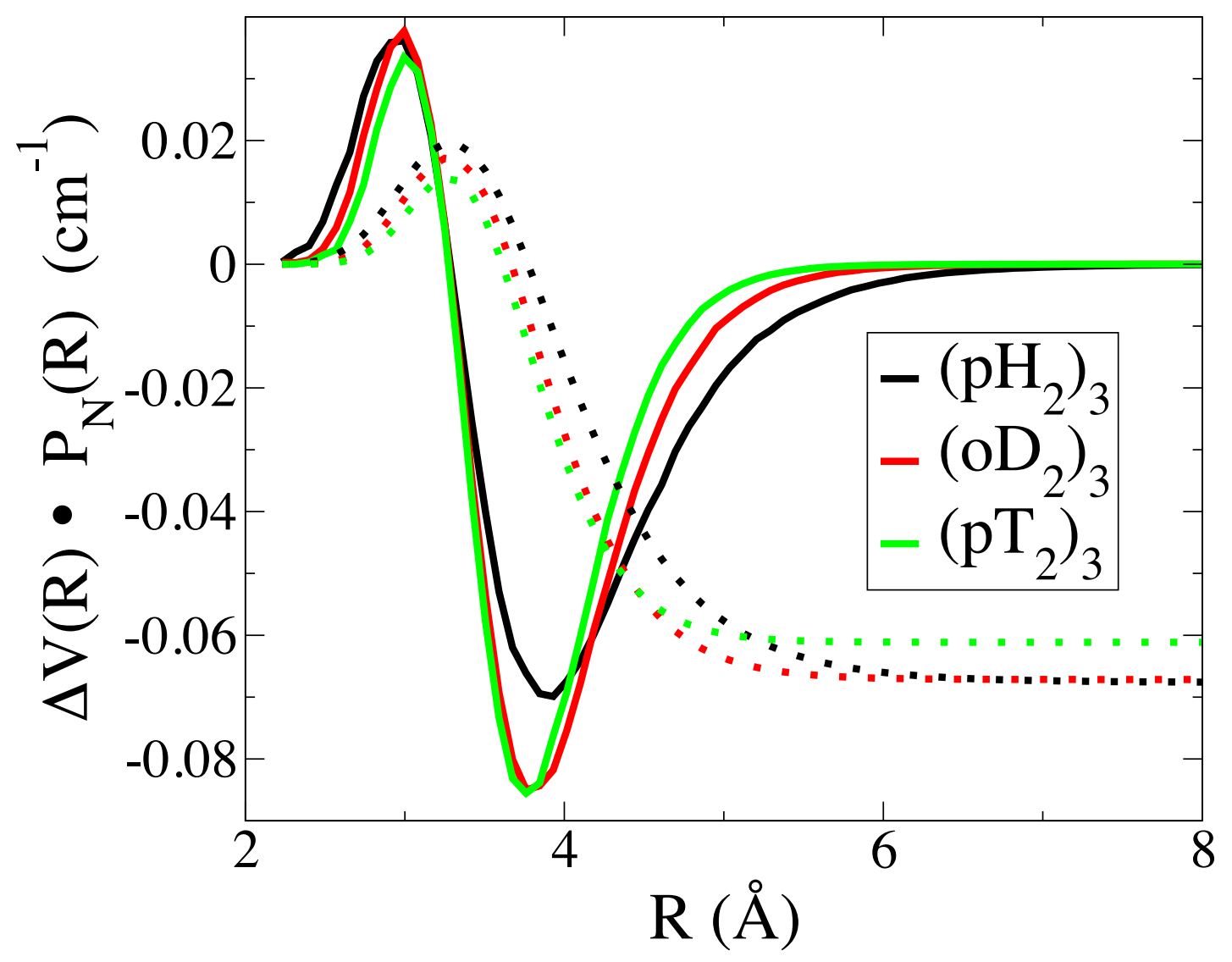

Figure 6: Solid lines: Integrand of the vibrational shift equation: the difference potential multiplied by the pair distribution function obtained from simulation, shown for the $\left(\mathrm{pH}_{2}\right)_{3}$, $\left(\mathrm{oD}_{2}\right)_{3}$, and $\left(\mathrm{pT}_{2}\right)_{3}$ trimers. Dotted lines: The cumulative sums of the integrand (scaled to fit the diagram) reflecting how the vibrational shifts change with intermolecular distance. 


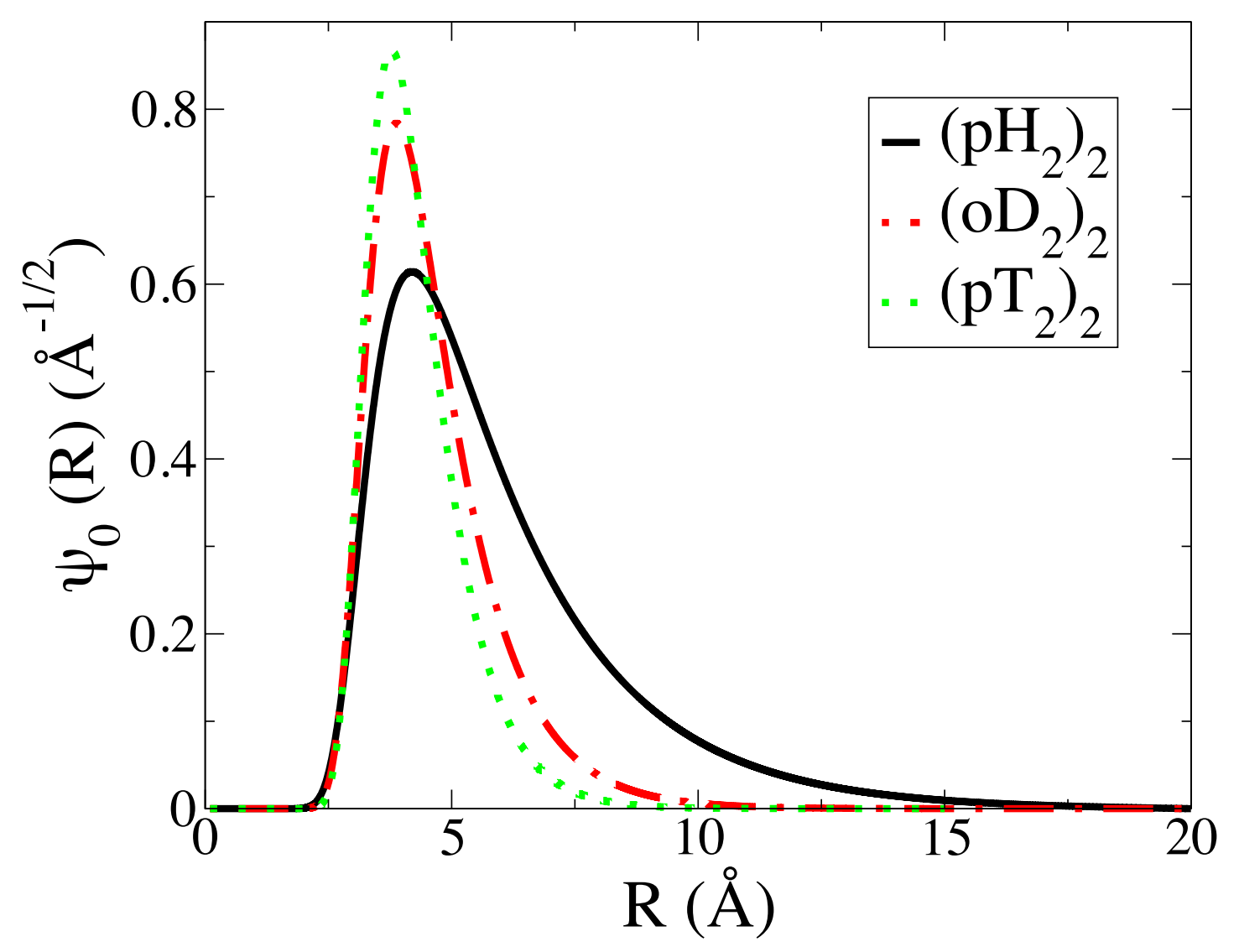

Figure 7: Ground state wavefunction of the $\left(\mathrm{pH}_{2}\right)_{2},\left(\mathrm{oD}_{2}\right)_{2}$, and $\left(\mathrm{pT}_{2}\right)_{2}$ dimers calculated by exact diagonalization using the H1-Ps. 

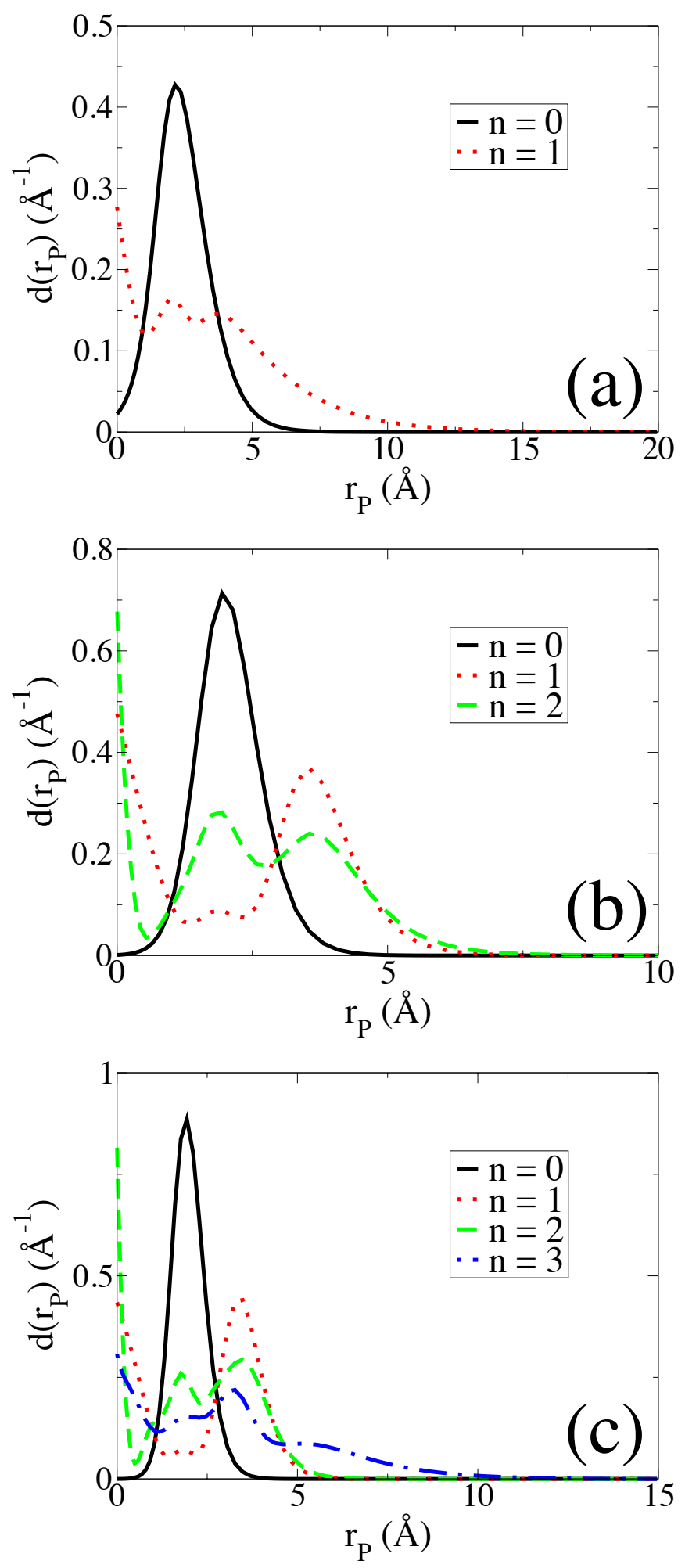

Figure 8: One-dimensional particle distribution for energy levels $E_{n}$ in Pekeris co-ordinates of (a) $\left(\mathrm{pH}_{2}\right)_{3}$, (b) $\left(\mathrm{oD}_{2}\right)_{3}$, and (c) $\left(\mathrm{pT}_{2}\right)_{3}$ trimers using the Lanczos diagonalization scheme. 